Published in final edited form as:

ACS Nano. 2020 September 22; 14(9): 11238-11253. doi:10.1021/acsnano.0c03109.

\title{
Cancer Cell Coating Nanoparticles for Optimal Tumor-Specific Cytokine Delivery
}

\author{
Antonio E. Barberio, \\ Department of Chemical Engineering, Massachusetts Institute of Technology, 183 Memorial \\ Drive, Cambridge, MA 02142, USA
}

\section{Sean G Smith,}

Department of Chemical Engineering, Massachusetts Institute of Technology, 183 Memorial

Drive, Cambridge, MA 02142, USA; Koch Institute for Integrative Cancer Research,

Massachusetts Institute of Technology, 500 Main Street, Cambridge, MA 02142, USA

\section{Santiago Correa, \\ Department of Biological Engineering, Massachusetts Institute of Technology, 21 Ames Street, Cambridge MA 02142, USA}

\section{Cathy Nguyen,}

Department of Biological Engineering, Massachusetts Institute of Technology, 21 Ames Street, Cambridge MA 02142, USA

\section{Bang Nhan, \\ Department of Chemistry, Wellesley College, 106 Central Street, Wellesley, MA 02481}

\section{Mariane Melo,}

Koch Institute for Integrative Cancer Research, Massachusetts Institute of Technology, 500 Main Street, Cambridge, MA 02142, USA

\section{Talar Tokatlian,}

Koch Institute for Integrative Cancer Research, Massachusetts Institute of Technology, 500 Main Street, Cambridge, MA 02142, USA

\section{Heikyung Suh,}

Koch Institute for Integrative Cancer Research, Massachusetts Institute of Technology, 500 Main Street, Cambridge, MA 02142, USA

Darrell J. Irvine*,

\footnotetext{
*CORRESPONDING AUTHORS: Paula T. Hammond: hammond@mit.edu, Darrell J. Irvine: djirvine@mit.edu. Author Contributions

AEB and PTH designed the experiments, analyzed the data and wrote the paper with significant aid from SGS. AEB, SGS, SC, CN, BTN, HS conducted the experiments. MM and HS designed and carried out the IL-12 manufacture. SGS and DJI provided advice and support throughout. PTH supervised the study. The manuscript was written through contributions of all authors. All authors have given approval to the final version of the manuscript.

Supporting Information Available

The supporting information includes calculations for number of IL-12 per NP, scIL-12 protein construct, additional microscopy images tracking subcellular localization, dose response curves for in vitro studies, in vitro tumor selectivity, temporal in vivo immune response studies, abscopal response studies, in vitro activity in additional models, flow cytometry gating strategies, and full detailed immune profiling results. This material is available free of charge via the internet at http://pubs.acs.org.
} 
Koch Institute for Integrative Cancer Research, Massachusetts Institute of Technology, 500 Main Street, Cambridge, MA 02142, USA; Department of Biological Engineering, Massachusetts Institute of Technology, 21 Ames Street, Cambridge MA 02142, USA; Department of Materials Science and Engineering, Massachusetts Institute of Technology, Cambridge, MA 02142, USA; Ragon Institute of Massachusetts General Hospital, Massachusetts Institute of Technology, Cambridge, MA 02139, USA; Howard Hughes Medical Institute, Chevy Chase, MD 20815, USA

\section{Paula T. Hammond ${ }^{\star}$}

Department of Chemical Engineering, Massachusetts Institute of Technology, 183 Memorial Drive, Cambridge, MA 02142, USA; Koch Institute for Integrative Cancer Research, Massachusetts Institute of Technology, 500 Main Street, Cambridge, MA 02142, USA; Institute for Soldier Nanotechnologies;

\section{Abstract}

Although cytokine therapy is an attractive strategy to build a more robust immune response in tumors, cytokines have faced clinical failures due to toxicity. In particular, interleukin-12 has shown great clinical promise but was limited in translation due to systemic toxicity. In this study, we demonstrate an enhanced ability to reduce toxicity without affecting efficacy of IL-12 therapy. We engineer the material properties of a NP to meet the enhanced demands for optimal cytokine delivery by using the layer-by-layer (LbL) approach. Importantly, using LbL, we demonstrate celllevel trafficking of NPs to preferentially localize to the cell's outer surface and act as a drug depot, which is required for optimal payload activity on neighboring cytokine membrane receptors. LbLNPs showed efficacy against a tumor challenge in both colorectal and ovarian tumors at doses that were not tolerated when administered carrier-free.

\section{Graphical Abstract}

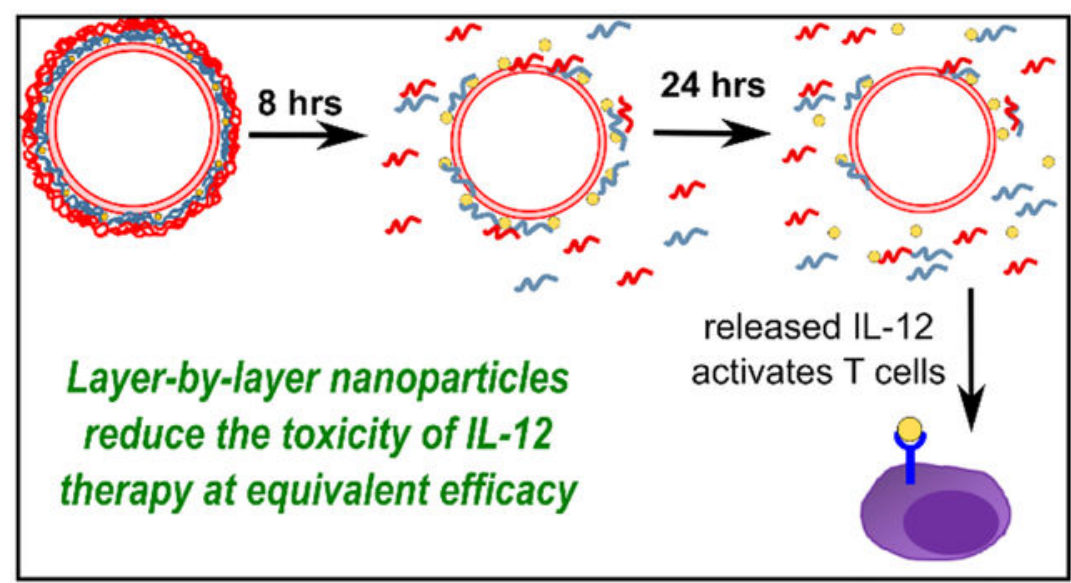

\section{Keywords}

nanomedicine; immuno-oncology; nanoparticle; drug delivery; layer-by-layer; cytokine

An effective immune response against cancer requires a complex series of steps, eloquently described as the cancer immunity cycle, ${ }^{1,2}$ which include tumor antigen release, dendritic 
cell priming of $\mathrm{T}$ cells in tumor-draining lymph nodes, and effector $\mathrm{T}$ cell homing to the tumor. These steps of the immune response are in a delicate balance and can either be arrested or driven forward by a variety of therapeutic approaches. Checkpoint inhibitors prevent the arrest of the cycle, which works well for some malignancies that have sufficient pre-existing tumor-specific immune populations present. For example, melanoma and lung cancer have shown some of the greatest success with these therapies; however, many epithelial tumors have been much more difficult to treat using immunotherapies to date. This is particularly true of cancers that are known to have a "cold" immune microenvironment such as advanced serous ovarian cancer, for which the concentration of prognostic leukocytes such as CD8+ T cells is low and the signaling cascade to elicit an immune response is greatly lowered or missing. ${ }^{3-5}$ Such "cold" tumor environments need additional therapies to drive the cycle forward, such as proinflammatory cytokines to increase the number and activity level of tumor specific immune cells both locally in the tumor microenvironment and systemically. One promising candidate for driving a proinflammatory response is interleukin-12 (IL-12). An extremely potent cytokine, IL-12 bridges innate and adaptive immunity and drives an antitumoral Th1 type immune response, mediated in large part by inducing the production of interferon- $\gamma$ (IFN- $\gamma$ ) that acts as the main downstream product of IL-12 signaling. ${ }^{6-9}$ IL-12 has been used in multiple preclinical models to great effect against various tumor types, ${ }^{10}$ which led to the initiation of multiple clinical trials of this cytokine for cancer therapy. ${ }^{11-13}$ Although initial trials found a well-tolerated dose, subsequent trials found high toxicity at the same dose with slightly altered schedule. It was found that these high, schedule-dependent toxicities were linked with the appearance of high IFN- $\gamma$ levels in blood plasma.

Recently, many delivery methods have been tested to improve IL-12 therapy. These methods have focused largely on local delivery techniques including microparticle delivery ${ }^{14-17}$ and chitosan co-formulations ${ }^{18-20}$ designed to keep IL-12 in the injected tumor; however, these methods require an accessible tumor to inject and cannot be translated to systemic delivery as is required for metastatic diseases that lack a main tumor such as ovarian cancer. Additional delivery methods have used gene therapy to induce IL-12 production in the tumor. ${ }^{21,22}$ Finally, simple nanoparticle delivery vehicles have been tested to deliver IL-12 protein ${ }^{23,24}$ relying on passive targeting, and notably lacking in sufficient toxicology studies demonstrating the reduction of toxic side effects for this potent therapy, a critical aspect of bringing the promise of IL-12 back to clinical relevance. All of these approaches lack the ability to spatio-temporally control the delivery of cytokine in the tumor environment from a systemically deliverable package; which is critical for meaningful success in the delivery of a highly potent and systemically toxic therapy such as IL-12 and other cytokines.

Optimally engineered NPs offer an evident opportunity to improve IL-12 therapy as it has been shown that localized IL-12 delivery is of great importance for a successful therapy. $18,20,25$ The use of a NP delivery system is particularly attractive because a properly designed NP therapy allows for the possibility of systemic delivery with limited off-target exposure of the IL-12 payload as well as concentration of IL-12 in the tumor microenvironment. Nevertheless, IL-12 poses design challenges for optimal NP delivery vehicles: 1) Cytokines are labile proteins and have been historically difficult to deliver using traditional encapsulation techniques; 2) NPs are often internalized by cells into endosomal 
compartments; however, IL-12 and other cytokines must engage external receptors to be effective and are rendered useless and often degraded upon internalization; 3) IL-12 is designed to be secreted and act locally in natural immune responses and is very toxic when allowed to circulate systemically, which necessitates a high degree of tumor association and display of IL-12 only within the tumor. Meeting all of these design criteria in the same NP delivery vehicle is paramount to the success of an IL-12 NP therapy.

Layer-by-layer (LbL) assembly is a simple, modular engineering approach for the surface modification of nanoparticles, which provides the means to address each of the design requirements defined above. LbL is a water-based, electrostatic method for layering polymer materials onto surfaces ${ }^{26,27}$ to generate a thin film of material that can modulate the material properties of the carrier. This NP design offers many advantages including multiple drug compartments with the potential for sequential cargo release, the ability to tailor surface chemistry with polymer layers to affect targeting and biodistribution, and improved pharmacokinetics. ${ }^{28-37}$ In particular, the LbL technique offers the ability to easily alter and screen for surface chemistries that can meet particular design criteria to address the many challenges presented by optimal IL-12 NP construction. In this work, an LbL particle construction approach was used to systematically engineer an optimized NP for IL-12 delivery against cancer. These particles were shown to meet each of the design criteria including 1) high loading and release of active IL-12,2) localization of NPs on the surface of tumor cells maintaining payload availability to membrane receptors, and 3) high association with tumor cells and decreased systemic exposure. These rationally engineered LbL NPs also crucially demonstrated greatly reduced toxicity of IL-12 therapy while maintaining activity in multiple murine cancer models, thus widening the therapeutic window for this potent therapy. Importantly, these LbL NPs showed efficacy in ovarian cancer, one of the more challenging tumor types to treat with immunotherapy as it often presents as a "cold" tumor that is difficult to treat using current approaches.

\section{Results/Discussion}

\section{Engineering layer-by-layer nanoparticles encapsulating IL-12}

A single chain version of IL-12 originally described by Lieschke et $a P^{8}$ was used for this work due to its enhanced stability and ease of production. This construct was engineered with a 6x His tag at the C-terminus for purification and encapsulation (Fig S1). The presence of the His tag was further utilized to attach the single chain IL-12 (scIL-12, IL-12) construct onto liposome surfaces via $\mathrm{Ni}$-His interactions with nickel bearing head groups from 1,2dioleoyl-sn-glycero-3-[(N-(5-amino-1-carboxypentyl)iminodiacetic acid)succinyl] (nickel salt) (DOGS-NTA (Ni)) on the liposome surface (Fig 1A). ${ }^{39}$ This attachment was performed using a simple overnight incubation of formed liposomes with IL-12, preserving the integrity and activity of the labile cytokine by avoiding liposome processing which requires heat, sonication and high pressure with the protein in solution. The IL-12 loaded liposomes were then further modified with a polyelectrolyte bilayer using the well-established LbL process, in which polymer layers of alternating charge are adsorbed to the particle surface and excess polymers removed using tangential flow filtration. ${ }^{32}$ These polymer layers serve two important purposes. First, the external anionic layer can be altered to tailor the targeting 
of the particles to particular cell types and cellular compartments. Second, the polymers act as a shield to the underlying IL-12, limiting its systemic exposure and toxicity. The initial cationic polymer layer on the particles was chosen to be poly-L-arginine (PLR) for its low toxicity and well-established use in LbL systems. ${ }^{35,40}$ For the external layer, both hyaluronic acid (HA) and poly-L-glutamic acid (PLE) were chosen to test based on their interactions with immune and cancer cells and dense negative charge ${ }^{41}$ (Fig 1A).

HA was chosen for its known ligand/receptor interaction with CD44 which has been utilized in the past to target cancers that overexpress CD44, such as triple negative breast cancer, non-small cell lung cancer and ovarian cancer. ${ }^{29,30,42-45}$ PLE was chosen based on its strong association and binding to ovarian cancer cells and immune cells of interest, as well as its subcellular localization to cell membranes. ${ }^{41}$ The resulting NPs were verified to be monodisperse and successful LbL constructs via cryoEM and dynamic light scattering (DLS) analysis (Fig 1B-D). LbL-NPs showed a number average radius of approximately $110 \mathrm{~nm}$ and an approximately $-60 \mathrm{mV}$ zeta potential for both surface chemistries irrespective of IL-12 loading. These particles showed efficient loading of cytokine, especially when compared to more traditional passive loading or loading via heparin binding of IL-12 16 in the layers of the particle (Fig 1E), with 90\% loading of IL-12 resulting in 13\% IL-12 by weight in the final formulation as compared to lipid weight. Using approximations based on lipid head group size, diameter, and monolayer thickness an estimation for number of lipid molecules in a unilamellar liposome can be generated and combined with the loading efficiency of IL-12 from Fig 1 to find that each LbL-NP contains approximately 50 IL-12 molecules (Calculation S1).

\section{Surface chemistry impacts LbL-NPs subcellular localization}

It is critical that the NPs associate primarily with tumor cell populations to prevent off-target toxicities; however, the receptor-mediated endocytosis achieved with typical selective binding to cells is not desirable for cytokine delivery, as it prevents the payload's access to membrane receptors. Thus, we hypothesize that NP delivery vehicles with extended cell surface membrane localization will improve IL-12 activity by allowing for greater interaction with its membrane receptor. To this end, fluorescence microscopy was used to probe the subcellular localization of both PLE and HA terminal layer LbL-NPs in target cells. Fluorescence microscopy showed that HA terminal layer NPs were internalized in both MC 38 colon carcinoma and HM-1 ovarian cancer cell lines by 24 hrs, while PLE terminal layer NPs remained bound to the surface membrane of the cells for extended periods without significant internalization in multiple cell lines (Fig 2, S2), consistent with recently reported work. ${ }^{41}$ Overall these results show promise for PLE as the terminal layer for IL-12-LbL-NPs as the increased cell surface localization on cancer cells has the potential to prolong IL-12 activity from the particles in the tumor microenvironment as compared to particles that are rapidly internalized.

\section{LbL NPs maintain IL-12 efficacy in vitro}

To evaluate the importance of extended membrane localization for IL-12 delivery via the NP carrier, the biological activity of the LbL-IL-12-NP constructs was probed and compared to free IL-12. To demonstrate biological activity, the IL-12 NPs' efficacy was tested in vitro by 
measuring their ability to stimulate an IFN- $\gamma$ response from primary splenocytes ${ }^{6}$ (Fig $3 \mathrm{~A}$ ). In an assay involving direct application to splenocyte culture, the IL-12 NPs showed less activity than an equivalent amount of free IL-12 as measured by the EC50 calculated from dose response curves (Fig 3C, S3). This result is expected, as binding and encapsulation of the cytokine on the NPs should reduce activity, and is indeed relied upon to ameliorate offtarget toxicity when the particles are delivered in vivo by preventing systemic IL-12 exposure. However, this experimental design does not fully mimic the tumor microenvironment the NPs experience in vivo upon delivery, which includes many more tumor cells than immune cells.

Having demonstrated activity directly on target immune cells, it is critical to show that the designed IL-12 NPs are able to maintain activity in the event that they are exposed to tumor cells before these target populations, as is likely in vivo. To this end, IL-12 NPs or free cytokine were first incubated with cancer cells before washing off media and adding splenocytes to the culture (Fig 3B). In this way, only therapies bound to cancer cells following the washing step, which mimics clearance, are still available to deliver cytokine to immune cells. PLE terminal layer LbL-IL-12-NPs (henceforth called PLE-IL-12-NPs) maintained the highest activity as measured by the lowest calculated EC50 value (Fig 3D, S3), outperforming both free IL-12 and HA terminal layer LbL-IL-12-NPs. Indeed even in this simulated worst-case scenario, PLE-IL-12-NPs maintained the activity of IL-12 in comparison to their direct activity on the target immune populations approximately $10 \mathrm{x}$ more than carrier free IL-12 and 2x more than readily internalized HA-IL-12-NPs (Fig 3E). This is likely due to the extended membrane localization of PLE-IL-12-NPs (Fig 2). Overall, these results indicated PLE-IL-12-NPs were the formulation most likely to maintain the activity of IL-12 within the tumor microenvironment, supporting our hypothesis that extended membrane localization is important for a cytokine delivery vehicle.

In addition, the identified PLE-IL-12-NPs were tested for their selectivity to tumor cells over immune cells as an initial in vitro test for viability of systemic delivery for such a NP system (Fig S4). It is critical that IL-12 be prevented from systemic activity for a successful IL-12 delivery strategy as off target toxicity is the most pronounced limit to IL-12 success in the clinic. To that end, it is paramount that an IL-12 delivery vehicle not only prevent systemic activity by preventing association with immune cells in circulation but also associate with tumor cells to concentrate NPs and their IL-12 payload in the tumor microenvironment. The described PLE-IL-12-NPs demonstrate this selectivity for tumor cell association as measured by flowcytometry using fluorescently tagged NPs dosed on a co-culture of splenocytes and MC38 tumor cells (Fig S4). These data demonstrate that the layering on the PLE-IL-12-NPs enhances NP uptake as compared to an unlayered construct and that NP association is highly ( $>92 \%)$ selective for tumor cells. These data together with the tumor cell membrane association show strong evidence that the described NP meets the design challenges of IL-12 delivery.

\section{IL-12 is exposed and released overtime from particle surface by erosion.}

Having demonstrated that the designed PLE-IL-12-NPs maintain pronounced activity in vitro, the mechanism of activity from the NPs was probed. For these studies PLE-IL-12-NPs 
were created with FRET pairing fluorophores (Fig 4A). The donor fluorophore (Cy3) of the FRET pair was bound to amines on 1,2-dioleoyl-sn-glycero-3-phosphoethanolamine (DOPE) on the liposome surface through NHS-ester chemistry. The acceptor fluorophore (Cy5) was bound to either free amines on IL-12 or the N terminal amine on PLR by NHSester chemistry. These NPs were then incubated at different solution conditions and samples were measured for FRET efficiency over a 72 hour period. The conditions tested were water (the storage buffer) and spent media (incubated on MC38 cells for at least 48 hours), which acts as an in vitro mimic to the conditions experienced in vivo, with the inclusion of serum and any factors produced by the tumor cells, such as enzymes. These FRET efficiencies were then normalized to a fully intact particle as defined by FRET efficiency at $\mathrm{t}=0$ and to a fully degraded (using Triton X-100) NP. FRET efficiency showed that the polymer layers undergo erosion of approximately $50 \%$ over an initial period of 8 hours followed by a slower erosion for the remainder of the study. IL-12 release showed a lag during the initial time while the polymer layers were still intact, with a matching burst between 8 and 24 hours, followed by a prolonged release over the remainder of the study (Fig 4B). These data demonstrate that the maintained IL-12 activity is achieved via surface erosion, in which the polymer layers first erode over an 8 hour period, subsequently revealing and releasing the underlying IL-12 layer (Fig 4C). Importantly, these data show that a majority of IL-12 is released in the first 24 hours, while the NPs are still localized to tumor surfaces (Fig 2), allowing for maintained IL-12 activity from the delivery vehicle as originally hypothesized.

Once full characterization and activity of the PLE-IL-12-NPs was established in vitro, their release and activity in vivo was probed to find an appropriate dosing schedule. C57BL/6 mice bearing MC38 tumors were treated intratumorally with PLE-IL-12-NPs (5 $\mu \mathrm{g} \mathrm{IL-12)}$ and sacrificed at different time points. Tumors were then homogenized and assessed for levels of delivered scIL-12, endogenous IL-12, and IFN- $\gamma$ by ELISA on tumor supernatants (Fig S5). Kinetic release of exogenously delivered scIL-12 matching the release found in Fig 4 was observed, followed by a peak response of endogenous IL-12 and a later peak of IFN$\gamma$. These data suggest an active immune response to PLE-IL-12-NPs over a seven day period, with the expected cascade of activation and interferon generation anticipated for an IL-12 therapy (Fig S5). These data indicate that a dosing schedule of at least weekly or more frequent dosing of PLE-IL-12-NPs may be required to maintain an active immune response in vivo.

\section{PLE-IL-12-NPs reduce the toxicity of IL-12 therapy}

The most critical aspect of any IL-12 therapy is the ability to control systemic activity and limit toxicity outside the tumor microenvironment as this has been the largest limitation of this potent class of cytokines in the clinic. PLE-IL-12-NPs were tested for their toxicity in healthy $\mathrm{C} 57 \mathrm{Bl} / 6$ mice which were dosed subcutaneously with $5 \mu \mathrm{g}$ doses of either the free cytokine or the PLE-IL-12-NP, as well as a group treated with $7.5 \mu \mathrm{g}$ of IL-12 in PLE-IL-12NPs, each dosed daily for 5 days. These groups were compared to PBS dosing and lipid concentration-matched PLE NPs without IL-12 as controls (Fig 5A). Mice given free IL-12 rapidly lost weight over the dosing period, losing approximately $10 \%$ of starting body weight by day 5 , indicating a highly toxic therapy. In contrast, mice showed little weight change compared to controls over the dosing period when given PLE-IL-12-NPs, even at 
1.5x the dosing of the free IL-12 (Fig 5B). These data demonstrate that PLE-IL-12-NPs reduce the systemic toxicity associated with IL-12 therapy.

As an additional test of toxicity, serum was collected from the subjects 3 hours after the final dose and analyzed for a panel of systemic inflammatory cytokines. This panel included IL-12 and IFN- $\gamma$ which are the direct downstream products of IL-12 signaling and most often associated with toxicity as well as CCL2 which acts as a chemoattractant for T cells, IL-10 which acts as the antithesis of IL-12 and is upregulated to slow down an inflammatory response, and TNF- $a$ which is an immune regulatory molecule. Carrier-free IL-12 therapy showed much higher levels of systemic cytokines IFN- $\gamma$ and IL-12 than PLE-IL-12-NPs, (Fig 5C), again indicating that PLE-IL-12-NPs are able to reduce the systemic toxicity of IL-12. Of note, when comparing the PLE-IL-12-NPs, specifically at the higher dose of 7.5 $\mu \mathrm{g}$, to free IL-12 many of the positive aspects of the systemic immune response remain engaged, such as chemoattraction of monocytes via CCL2, while the highly toxic IFN- $\gamma$ is drastically reduced in systemic circulation by the PLE NP carrier even at the higher dose. This demonstrated reduction of systemic toxicity is critical for a successful IL-12 therapy.

\section{PLE-IL-12-NPs maintain efficacy of IL-12 therapy in multiple tumor models}

To determine whether safer IL-12 delivery from the described NP carrier continued to provide antitumor efficacy, PLE-IL-12-NPs were tested for their ability to slow tumor growth and prolong survival in two flank tumor models, MC38 colon cancer and the more difficult to treat HM-1 ovarian cancer, as a test of anti-tumor response. As an initial test, MC38 cells were implanted subcutaneously in C57BL/6 mice and vascularized tumors were allowed to form (6 days). Once tumors were established, mice were treated intratumorally with both free IL-12 and PLE-IL-12-NPs and compared to appropriate controls (PBS and PLE NPs) (Fig 6A). Mice were monitored for tumor response both by growth of tumor size (Fig 6B, C) and overall survival (Fig 6D). Subjects receiving $5 \mu \mathrm{g}$ weekly injections of both carrier-free IL-12 and PLE-IL-12-NPs showed slowed tumor growth and prolonged survival compared to both the PBS and PLE NP control groups in a statistically significant manner by the thirteenth day of tumor growth. Importantly, subjects treated with free IL-12 and PLE-IL-12-NPs showed no differences in therapeutic response from each other. Also, increasing the dose and dosing frequency of PLE-IL-12-NPs enabled a further improvement in tumor responses, as demonstrated with $7.5 \mu \mathrm{g}$ dosing of the PLE-IL-12-NPs or increased $2 \mathrm{x}$ weekly frequency of dosing; both of these conditions elicited slower tumor growth and a statistically significant survival benefit as compared to $5 \mu \mathrm{g}$ weekly doses of IL-12 in both the PLE NP encapsulated form and free drug (Fig 6B-D). These data together with the previously discussed reduction in toxicity demonstrate a widening of the therapeutic window for this potent yet toxic therapy.

In addition to the described single tumor efficacy tests, the ability of PLE-IL-12-NPs to provoke an abscopal immune response was tested in a two flank model (Fig S6). Mice were inoculated with MC38 tumor cells subcutaneously on both flanks, with a smaller (5x less inoculant) tumor inoculation on the untreated (left) flank. Tumors on the right flank were treated with PBS, PLE-IL-12-NPs, or free IL-12 at $5 \mu \mathrm{g}$ weekly doses and both tumors were monitored for response. Treated tumors responded equally well to PLE-IL-12-NPs and free 
IL-12; however, the abscopal untreated tumor growth was slowed by both PLE-IL-12-NPs and free IL-12 with a slightly better (statistically insignificant) response to the free IL-12. This is likely due to the greater systemic activity of the carrier-free treatment shown in (Fig 5). Notably there is still a trend in abscopal response to the PLE-IL-12-NPs compared to controls suggesting a systemic immune response against the tumor. Coupled with reduced toxicity (Fig 5) the equivalent efficacy of PLE-IL-12-NPs to carrier-free IL-12 demonstrates an improvement in IL-12 therapy using the described PLE-IL-12-NPs.

Showing efficacy in the MC38 model is an excellent indicator of the viability of the described PLE-IL-12-NPs as an effective cytokine delivery vehicle. However, the MC38 cell line is known to be an immune infiltrated or "hot" tumor model. Ovarian tumors have proven much more difficult in their response to immunotherapies. ${ }^{3-5}$ As a further measure of IL-12 efficacy and test for viability of cytokine delivery via PLE-NPs, their efficacy in HM-1 tumors (murine ovarian tumors known to be heavily infiltrated with myeloid derived suppressor cells and poorly infiltrated with T-cells) was tested. ${ }^{47,48}$ This tumor model also showed improvement in tumor responses both by slowing of tumor growth (Fig $6 \mathrm{E}, \mathrm{F}$ ) and survival (Fig 6G) when treated with IL-12, with PLE-IL-12-NPs achieving equivalent efficacy to the more toxic carrier-free therapy. This model was further tested using the same in vitro assays described for the MC38 model in Fig 3. Similar responses in the in vitro efficacy tests in both the splenocyte only and co-culture models (Fig S7) were obtained with HM-1 cells as well as 4T1 breast cancer cells as compared to the MC38 experiments, establishing PLE-IL-12-NPs as a diversely applicable therapeutic strategy. These data together with toxicity reduction suggest that PLE-IL-12-NPs show promise for treatment of multiple tumor types, including those traditionally less responsive to immunotherapy such as ovarian cancer.

Ovarian cancer generally has not benefitted from the clinically available checkpoint inhibitors, and the exploration of inflammatory molecules such as IL-12 and some other cytokines as a supplement to checkpoint inhibiters has been limited by toxicity concerns. Ovarian cancer usually does not present with a single injectable tumor but instead with many metastatic nodules spread throughout the abdomen, making intratumoral treatment an impractical option clinically. The delivery system described in the current study demonstrates promise for systemic or intraperitoneal application of toxic cytokines in these difficult tumors; polymer layers not only reduce the toxicity of the cytokine as described herein, but also may provide a significant targeting effect to ovarian tumors in vivo that can effectively concentrate the delivery of cytokines to the tumor nodules. ${ }^{41}$ Indeed, systemic immunotherapy via cytokine delivery offers promise for widely disseminated metastatic disease such as ovarian cancer as developing a local immune response in some tumors can lead to systemic immunity against the cancer at other metastatic sites. Our PLE-IL-12-NPs demonstrate this systemic effect in their efficacy against abscopal tumors. Expanding the applications of the described PLE-IL-12-NPs to systemic delivery poses the promise of bringing immunotherapy advancements to the ovarian treatment space.

As a further test of the LbL-NP's ability to improve IL-12 therapy, PLE-IL-12-NPs were tested against carrier-free delivery for both toxicity and efficacy at greatly increased dosing levels to further demonstrate the described widening of the therapeutic window. For these 
tests, subjects were treated with PLE-IL-12-NP doses increased to $25 \mu \mathrm{g}$ (5x the normal dose from Fig 6) and $50 \mu \mathrm{g}$ (10x the normal dose) given twice weekly (as opposed to weekly dosing in Fig 6) for five doses and compared to $25 \mu \mathrm{g}$ and $50 \mu \mathrm{g}$ free IL-12 given twice weekly. Tumor growth and survival was analyzed for efficacy and weight change over the dosing period and serum cytokine levels after the first and last treatments were analyzed for toxicity (Fig 7A). Groups treated with free IL-12 lost weight after each dose, with the $50 \mu \mathrm{g}$ treated mice losing significant weight ( $10 \%$ body weight) following the first treatment, thus reaching the level of weight loss that exceeds that of the maximum tolerated dose as defined for these studies, evidencing a highly toxic therapy. Conversely, groups treated with PLEIL-12-NPs continued to gain weight with the controls (Fig 7B) at the same dosing levels. In addition, both IL-12 and IFN- $\gamma$ levels in the serum were higher in the free IL-12 treated mice after the first and last dose, particularly at the higher dosing level of $50 \mu \mathrm{g}$. At this dose the free IL-12 treated groups showed significant differences to the PLE-IL-12-NP groups throughout the dosing period, especially in the toxic systemic IFN- $\gamma$ levels (Fig 7C), again demonstrating the systemic toxicity of free cytokine in these subjects. These data suggest that PLE-IL-12-NPs greatly reduce the toxicity of IL-12 treatment even at very high doses. Taking this study further, the group treated with the $25 \mu \mathrm{g}$ dosing level of the PLE-IL-12NPs showed equivalent efficacy compared to the group dosed with free IL-12 in terms of both tumor growth arrest and survival (Fig 7D, E). Moreover, the group dosed with the $50 \mu \mathrm{g}$ PLE-IL-12-NPs showed significantly longer survival compared to the group dosed with 25 $\mu \mathrm{g}$ of free IL-12, which was the highest well-tolerated dose of free cytokine tested, at reduced toxicity. Furthermore, the PLE-IL-12-NP $(50 \mu \mathrm{g})$ resulted in a cured mouse which rejected a rechallenge with $1 \times 10^{6}$ tumor cells 85 days after treatment, indicating a memory response. Combining these data with Fig 5, the dose response achieved by IL-12 therapy both in free and PLE NP form is evident (Fig 7F). These data show that the rationally engineered PLE-IL-12-NPs make a significant improvement for IL-12 therapy by allowing for increased dosing levels with reduced toxicity over what is achievable with carrier-free cytokine with no loss in efficacy of the IL-12 treatment. The ability to effectively treat epithelial cancers including ovarian cancers at tolerated doses with proinflammatory cytokines such as IL-12 is critical in potentiating the promise of immunotherapies in these tumors, as these therapies are capable of driving immune infiltration and activity in these difficult to treat tumors.

One limitation of the current study is that it relies on an intratumoral approach in in vivo studies to deliver the therapeutic. However, overcoming IL-12 based toxicity even from a local injection has remained a challenge clinically and preclinically with several investigations taking a local delivery approach still failing to reduce systemic toxicity when the IL-12 is given at therapeutically relevant dose. Indeed, even in the current study, locally delivered free IL-12 showed significant toxicity at relevant doses. Therefore, even though the reduction in toxicity shown in the current study is thus far only demonstrated for intratumoral administration, this result still stands as a relevant advance for IL-12 delivery. Nonetheless, ongoing and future work will explore the application of this delivery system for systemic administration, focusing on ovarian tumor models to bring the promise of immunotherapy to this difficult to treat malignancy. Indeed the in vitro work here shows promise for a successful systemic cytokine delivery platform. Firstly, the described PLE- 
IL-12-NPs showed selective association with tumor cells both in this work (Fig S4) and previous studies, ${ }^{41}$ which is critical for concentrating NPs and payload in the tumor microenvironment. In addition, mechanistic studies showed the majority of the IL-12 remains attached to the particle for greater than 8 hours (Fig 4), which is longer than similar actively targeted NPs take to concentrate in the tumor microenvironment upon systemic delivery. This delayed IL-12 release is critical for potential success in systemic delivery as it prevents off-tissue exposure of potent cytokine payloads. Equally important, the IL-12 payload is rapidly released thereafter while the NPs remain localized to the cell surfaces, maintaining the cytokine payload activity once in the tumor microenvironment. Taken together, the in vitro characterizations of IL-12 loading (Fig 1), subcellular localization (Fig 2), tumor cell association selectivity (Fig S4), biological activity (Fig 3), and kinetic release of IL-12 (Fig 4) demonstrate a NP platform that meets all the criteria for a successful systemically deliverable cytokine therapy.

\section{PLE-IL-12-NPs enhance lymphocyte activity in tumors and tumor draining lymph nodes}

After showing that the described PLE-IL-12-NPs were capable of reducing toxicity with comparable anti-tumor efficacy compared to free cytokine, flowcytometry was used to probe the immunological mechanisms of the treatments in the MC38 model. As described above, vascularized tumors were allowed to establish before starting therapy (6 days). Tumors were then treated intratumorally with biweekly doses of $5 \mu \mathrm{g}$ IL-12 either in PLE-IL-12-NP form or carrier-free, PLE-NPs with matched lipid dose, or PBS. Tumors, spleens, and tumor draining lymph nodes (TDLNs) were prepared for flowcytometry 24 hours after the third dose which was chosen as an endpoint based on significant differences in tumor sizes occurring between treatment groups at that time as shown in Fig 6. Flowcytometry was used to distinguish different cell populations in the isolated tissues (gating strategy Fig S8, all data Fig S9).

As expected, both IL-12 therapies caused large shifts in lymphocyte populations indicative of a more active antitumor immune response in the tumor microenvironment. The main impact from IL-12 therapy both in carrier-free and PLE-IL-12-NPs was a shift towards higher CD8+ T cell populations in the tumor (Fig 8A). This is evidenced not only by a higher CD8+ fraction of $\mathrm{T}$ cells in the tumor, but also by a higher ratio of CD8+/CD4+ $\mathrm{T}$ cells in the tumor (Fig 8E). Moreover, IL-12 therapy yielded more active CD8+ T cells in the tumor, as measured by degranulation markers (CD69+) (Fig 8B,E). Importantly, the PLE-IL-12-NPs showed no difference in CD8+ T cell or degranulated CD8+ T cell populations compared to free IL-12, indicating the same gains in immune activation state in the tumor microenvironment as the free drug, despite the therapy's encapsulation. These $\mathrm{T}$ cell shifts are expected from an IL-12 therapy and indicative of a shift toward a more active, antitumoral Th1 type immune response in the tumor. In addition, the monocyte populations in the tumor undergo a shift from more monocytic (Ly6C+) to more granulocytic (Ly6G+) (Fig S9) indicating a polarization of monocytes to neutrophils and a more active local immune response.

In addition to these intratumoral T cell shifts, the IL-12 therapies showed a shift towards greater APC migration to TDLNs. Both DC and macrophage populations were increased in 
TDLNs with both the PLE-IL-12-NPs and free IL-12 (Fig 8C,D,F). This migration of APCs to the TDLNs is again indicative of a more active immune response to the tumor. Migration of APCs to the lymph node is an expected step in the immunity cycle in response to an IL-12 therapy driving the cycle forward. ${ }^{1,2}$

Little changes were seen in the spleen of treated mice, with the sole differences between IL-12 therapy and the control being an increase in neutrophil populations and a slight decrease in CD45+ leukocyte fractions in the treated groups (Fig S9). Taken together, these immune population changes upon treatment with IL-12 both within PLE-IL-12-NPs and carrier-free show a shift towards a more active and antitumoral Th1 type immune response in the tumor microenvironment and local lymphoid tissue, as is expected from an IL-12 therapy. These data demonstrate that PLE-IL-12-NPs are able to trigger a potent immune response and drive the cancer immunity cycle forward equivalently to carrier free IL-12 at significantly reduced toxicity.

Additional leukocyte population shifts indicate a shift towards a less suppressive immune microenvironment with IL-12 therapy. The PLE-IL-12-NP treatment showed a reduced number of regulatory $\mathrm{T}$ cells (Tregs) in the tumor coupled with a higher ratio of CD8/Treg cells in the TDLN. In addition, PLE-IL-12-NPs showed a trend towards reduced expression of PD-L1 (CD274) on CD45+ cells as compared to free IL-12. This difference is mainly from macrophage expression differences in PD-L1, as there is little difference in the DC population PD-L1 expression (Fig S9). Together, these data indicate that PLE-IL-12-NPs are capable of reducing the immune suppressive tumor microenvironment, particularly in the macrophage population, potentially beyond what is achievable with free cytokine, which could prove to be a potentially helpful enhancement in treatment.

\section{Conclusions}

In this work we have shown the rational engineering of an LbL NP delivery vehicle for the potent but toxic cytokine IL-12. The PLE-IL-12-NP met all of the key design criteria for cytokine delivery including efficiently encapsulating and releasing the cytokine, maintaining cytokine activity on external cell receptors, selectively interacting with tumor cells, achieving anti-tumor efficacy in multiple tumor models at reduced systemic toxicity compared to carrier free cytokine, and engaging local and systemic immunity. We have demonstrated that the described PLE-IL-12-NPs have a pronounced ability to encapsulate IL-12, showing a $90 \%$ encapsulation at $13 \%$ by weight in stable particles (Fig 1). We have shown that PLE-IL-12-NPs maintain the efficacy of IL-12 in its ability to stimulate IFN- $\gamma$ production from target cells, and indeed exceed the activity of free IL-12 in a worst-case tumor mimic in vitro model, likely due to the distinctive ability of PLE-IL-12-NPs to localize on tumor cell membranes and act as a drug depot (Fig 2,3,4). We have also demonstrated that PLE-IL-12-NPs are capable of releasing active IL-12 and triggering an immune response in vivo, and that this immune response is capable of slowing tumor growth in multiple cancer models as a monotherapy, notably including more difficult to treat ovarian cancer (Fig 5,6). 
Most importantly for IL-12 therapy, we have demonstrated in multiple studies the ability of the described PLE-IL-12-NPs to reduce the systemic toxicity of IL-12 at multiple schedules and doses. Indeed, we have demonstrated that the PLE-IL-12-NPs offer a safer therapy, even at higher doses than the free cytokine (Fig 5,7), which in turn yields a more effective therapy due to the ability to access higher dosing levels as compared to carrier free cytokine with the two delivery strategies showing equivalent efficacy at equivalent dosing. This is of utmost importance for any IL-12 therapy as toxicity has been the largest factor in preventing IL-12 from progressing in the clinic as evidenced by the severe toxicity, including two deaths in previous trials. $^{11-13}$

Finally, while these PLE-IL-12-NPs make use of the potent cytokine IL-12, no IL-12 specific techniques are used for their generation, making them adaptable to many other cytokines or proteins of interest for delivery. Moving forward, these particles can be utilized to deliver any protein with an appropriate binding ligand, or indeed a combination of such molecules. The general design is also easily adaptable to many other conjugation strategies for linking the cytokines to liposomes that can be used to modulate the release rate of delivered proteins. Accordingly, another advantage of the described LbL NP system is easy adaptability to combination therapy. Additional therapeutics can be added to the particle in multiple ways, including adding additional cytokines on the liposome surface, adding small molecules to the liposome core as described previously, ${ }^{29,36}$ or including therapeutics such as siRNA within the layers. These particles can also be used to incorporate checkpoint inhibitors such as anti PD-1 or anti CTLA4 in a similar manner to cytokines on the liposome surface or attached to the layers. These combinations could prove to be most effective, because for many malignancies, a combination of immunotherapies is viewed as the most promising path forward. ${ }^{49-51}$ As this approach progresses, the need to deliver combinations of immunotherapeutics in precise ratios and schedules ${ }^{52}$ will also need to be controlled another potential benefit of using the described LbL-NPs, which can be designed to stage release of combination therapies. ${ }^{31}$

\section{Methods/Experimental}

\section{Materials}

1,2-dioleoyl-sn-glycero-3-[(N-(5-amino-1-carboxypentyl)iminodiacetic acid)succinyl] (nickel salt) (DGS-NTA (Ni)), 1,2-distearoyl-sn-glycero-3-phosphocholine (DSPC), Cholesterol, and 1-palmitoyl-2-oleoyl-sn-glycero-3-phospho-(1'-rac-glycerol) (sodium salt) (POPG) 1,2-dioleoyl-sn-glycero-3-phosphoethanolamine (DOPE) were purchased from Avanti Polar Lipids and used without modification. PLR and PLE were purchased from Alamanda Polymers and used without modification. Single chain IL-12 was produced in house from HEK-293 cells.

\section{Protein Synthesis and Purification}

Single chain IL-12 sequence ${ }^{38}$ was synthesized as a genomic block (Integrated DNA Technologies) and cloned into gWIZ expression vector (Genlantis). Plasmids were transiently transfected into Expi293 cells (ThermoFisher Scientific). After 5 days, cell culture supernatants were collected and protein was purified in an ÄKTA pure 
chromatography system using HiTrap HP Niquel sepharose affinity column, followed by size exclusion using Superdex 200 Increase 10/300 GL column (GE Healthcare Life Sciences). Endotoxin levels in purified protein was measured using Endosafe Nexgen-PTS system (Charles River) and assured to be $<5 \mathrm{EU} / \mathrm{mg}$ protein.

\section{Particle Formulation and Characterization}

LbL nanoparticle assembly was performed as described previously ${ }^{32}$ with minor modifications. Briefly, liposomes were prepared by the rehydration/extrusion method. A lipid solution containing 5\% DGS-NTA (Ni), 65\% DSPC, 23.9\% Cholesterol, and 6.1\% POPG by mole in chloroform was dried at $20 \mathrm{mbar}$ for $1 \mathrm{hr}$ by rotovap and desiccated under vacuum overnight. The lipid film was then reconstituted in PBS to a concentration of 1 $\mathrm{mg} / \mathrm{mL}$ under sonication at $65^{\circ} \mathrm{C}$ for 30 minutes. Rehydrated liposomes were extruded through $50 \mathrm{~nm}$ filters (Whatman) using Avestin Liposofast-50 pressure driven extruder at $65^{\circ} \mathrm{C}$ until they reached a size of appx $60 \mathrm{~nm}$ as measured by number average diameter by DLS (Malvern ZS90). Single chain IL-12 was added to liposomes in a 28:1 Ni:His ratio by mole overnight at 4C. Particle buffer was switched to water by tangential flow filtration (TFF) by $5 x$ washing through a $100 \mathrm{kDa}$ membrane (Spectrum Labs). Particles were added to a bath of PLR solution in glass vial under sonication at 0.1 weight equivalent of polymer compared to lipid and allowed to equilibrate on ice for 1-2 hours. Excess polymer was purified by TFF through a $100 \mathrm{kDa}$ membrane (Spectrum Labs) and characterized for size and charge by DLS. Similarly, for terminal layer polyanion, particles were then added to a bath of polymer in glass vial under sonication at 1 weight equivalent of polymer compared to lipid and allowed to equilibrate on ice for 1-2 hours. Particles were purified by TFF and characterized for size and charge by DLS.

\section{Encapsulation characterization}

Encapsulation of scIL-12 in particles at various stages of the particle manufacture process was characterized by breaking up particles in 1\% triton-100 (Sigma), 0.1\% BSA (Sigma) under vortex for $1 \mathrm{~min}$. IL-12 content was then measured by ELISA (Peprotech) and compared to initial amount of IL-12 added to the NPs (measured by nanodrop at time of addition) for encapsulation efficiency. Final particle lipid concentration was measured by Stewart assay ${ }^{53}$ using chloroform dissolved lipid mixture from liposome formulation to produce a standard curve and weight percent encapsulation was calculated using IL-12 and lipid concentrations (mg IL-12/(mg lipid+mg IL-12)).

\section{Flowcytometry}

Antibodies used for immunostaining were against CD69 (biolegend 104545), CD25 (biolegend 102041), NK-1.1 (biolegend 108753), CD3 (biolegend 100232), CD4 (biolegend 100423), CD8a (BD biosciences 566410), FoxP3 (biolegend 126404), CD45 (biolegend 103112), Ly-6C (biolegend 128032), Ly-6G (biolegend 127633), CD274 (biolegend 124331), F4/80 (biolegend 123110), CD11c (BD biosciences 566504), CD11b (biolegend 101217), CD86 (biolegend 105037) CD103 (biolegend 562722). FoxP3 intracellular staining was carried out using FoxP3 intracellular staining kit (Thermo 00-5523-00) following manufacture protocol. Immunostained cells were run on an LSR Fortessa HTS with FACSDIVA software and analyzed using FlowJo V10.5.3. 


\section{Fluorescence Imaging}

Chambered cover slips (LabTek) were coated with $50 \mu \mathrm{g} / \mathrm{mL}$ rat tail collagen (corning).

Cells were seeded on coverslips at 5000 cells per well and allowed to establish for 24 hours. Cells were treated at different time points at $6.2 \mu \mathrm{g} / \mathrm{mL}$ particles. Fluorescent core carboxy modified latex particles (Thermo Fisher F8803) layered with PLR and indicated terminal layer polyanions were used in place of scIL-12 liposome cores for visualization. Cells were incubated for 4 or 24 hours after particle treatment. Cells were fixed with $4 \%$ formaldehyde for 15 minutes and stained with wheat germ agglutinin-AF647. Cells were then fixed again for 2 minutes and permeabilized with $0.2 \%$ triton $\mathrm{x}-100$ and stained with Hoechst solution at $1.25 \mu \mathrm{g} / \mathrm{mL}$. After staining, wells were protected with Vectashield and imaged on an Applied Precision DeltaVision Spectris Imaging System with Softworx deconvolution software. Images were further analyzed in FIJI.

\section{Cell Culture}

MC38 cells were a gift from the laboratory of Darrell Irvine. HM-1 cells were acquired through Riken BRC. Cells were cultured in DMEM or a-MEM respectively, supplemented with $10 \% \mathrm{FBS}$ and penicillin/streptomycin or as recommended by the supplier in a $5 \% \mathrm{CO}_{2}$ humidified atmosphere at 37C. All cell lines were murine pathogen tested and confirmed mycoplasma negative by Lonza MycoAlert ${ }^{\mathrm{TM}}$ Mycoplasma Detection Kit.

\section{In vitro activity experiments}

Constructed scIL-12 LbL NPs were tested for bioactivity by ability to stimulate an IFN- $\gamma$ response. Briefly, splenocytes were isolated from appropriate background strain mice (based on the tumor cell line being used; MC38 paired with $\mathrm{C} 57 \mathrm{~B} 1 / 6$ mice and $\mathrm{HM}-1$ paired with B6C3F1) by pushing spleens through $70 \mu \mathrm{m}$ strainers, lysing red blood cells via ACK lysis buffer and resuspending splenocytes in RPMI containing 10\% FBS, $1 \%$ penicillin/ streptomycin, $1 \%$ sodium pyruvate, and $0.0005 \% \beta$-mercaptoethanol. Splenocytes were cultured for 24 hours or less to prevent changes in populations from culture. Splenocytes were either dosed with varying doses of scIL-12 in particles or soluble form or added to cancer cell cultures that had been dosed for 6 hrs previously with varying doses of scIL-12 in particle or soluble form for 18 hours. Supernatants were then tested for IFN- $\gamma$ content by ELISA (peprotech). Data were analyzed in Graph Pad PRISM 5 to find EC50 values based on dose response curves.

\section{Tumor selectivity studies}

MC38 cells were seeded in 96 well plates at 50000 cells per well and cultured for 24 hours. Splenocytes were harvested similar to in vitro activity experiments and added to wells (1:1 ratio with MC38 cells) containing subcultured MC38 cells for a total volume of $90 \mu$. Wells were then dosed with $10 \mu \mathrm{l}$ of $0.1 \mathrm{mg} / \mathrm{mL}$ or $0.01 \mathrm{mg} / \mathrm{mL}$ Cy5 NPs at varying timepoints. Cy5 NPs were made by addition of 5\% DOPE by mol replacing 5\% by mol DSPC in original liposome formulation. These liposomes were then tagged with sulfo-Cy5 NHS ester (Lumiprobe 13320) following manufacture protocol. Briefly, 8 fold molar excess of dye was added to NP solution titrated to $\mathrm{pH} 8.5$ and allowed to react at $4 \mathrm{C}$ overnight under agitation. Excess dye was purified off the NPs using TFF. NP solution was washed until the permeate 
from TFF showed $<5 \%$ fluorescence as compared to the start of purification. IL-12 and polymer layering was then carried out similar to previous formulations. After NP incubation cultures were processed and stained for live/healthy cells (Biolegend 423114) and CD45 (BD 564279). Flowcytometry was carried out similar to previous description.

\section{FRET studies}

NPs were formulated similar to the above description. For these studies liposomes were made following the same protocol as described previously with the exception that 5\% DOPE was added and DSPC mol\% was reduced to $60 \%$. Once liposomes were extruded and buffer exchanged to water, Sulfo-Cy3 NHS-ester (Lumiprobe 11320) was added to the liposome surface following manufacture protocol for NHS-ester amine reaction with DOPE head groups. Briefly, 8 fold molar excess of dye was added to NP solution titrated to $\mathrm{pH} 8.5$ and allowed to react at $4 \mathrm{C}$ overnight under agitation. Excess dye was purified off the NPs using TFF. NP solution was washed until the permeate from TFF showed $<5 \%$ fluorescence as compared to the start of purification. IL-12 was then added to the liposome surface as described previously. For IL-12 release studies Sulfo-Cy5 NHS ester was added to the IL-12 similar to Sulfo-Cy3 NHS ester addition to DOPE described above. NPs were then layered with polymer layers similar to previous experiments. For NP erosion FRET particles SulfoCy5 NHS ester was added to PLR similar to addition to IL-12. NPs were made in two groups, IL-12 tagged and PLR tagged. Each group contained NPs with only Sulfo-Cy3 on the liposome surface, NPs with only Sulfo-Cy5 either on the IL-12 or PLR respectively, and FRET pairing NPs with both dyes present. NPs were then added at $10 \%$ by volume to different media conditions. Media conditions tested included water (storage condition) and cell media that had been incubated with active cultures of MC38 cells for no less than 48 hours (spent media) as a mimic of an in vivo environment. Samples were taken at 1, 2, 4, 8, 24, 48 and 72 hour timepoints and measured for fluorescence. Two measurements were taken, 1) 540 excitation and 570 emission reflecting excitation and emission of Cy3 and 2) 540 excitation and 680 emission reflecting excitation of Cy3 and emission of Cy5. FRET efficiency was then calculated based on equation 1 .

$$
\text { FRET efficiency }=\frac{D_{D}-\text { Fret }_{D}}{D_{D}}
$$

$D_{D}=$ fluorescence intensity of NP with donor fluorophore only in the donor channel

Fret $_{D}=$ fluorescence intensity of NP with both fret fluorophores in the donor channel

FRET efficiency was normalized to $100 \%=$ FRET efficiency at $\mathrm{t}=0$ and $0 \%=$ FRET efficiency of NPs degraded with triton $\mathrm{x}-100$ similar to encapsulation experiments described above.

\section{Animal Studies.}

All animal experiments were approved by the Massachusetts Institute of Technology Committee on Animal Care (CAC) and were conducted under the oversight of the Division of Comparative Medicine (DCM). 


\section{In vivo toxicity tests}

To test toxicity, C57B1/6 mice (Taconic) were injected subcutaneously (healthy mice) on the flank with varying doses as indicated of PLE-IL-12-NPs, lipid dose matched LbL-NPs without IL-12, dose matched soluble IL-12, or PBS and monitored daily for weight change. Serum was collected after 5 days and assayed using multiplex inflammatory cytokine assay (done by Abcam). (PLE IL-12 NPs 5 $\mu \mathrm{g} n=5, \mathrm{n}=3$ all other groups)

\section{In vivo efficacy tests}

C57B1/6 (Taconic) or B6C3F1 (Jackson Labs) mice as appropriate for tumor type were injected subcutaneously with 5E05 (MC38) or 1E06 (HM-1) cells in 1:1 PBS:Matrigel (Corning) mixture. Tumors were allowed to establish for 6 days prior to treatment. Mice were treated intratumorally with scIL-12 LbL-NPs, lipid dose matched LbL-NPs without IL-12, dose matched soluble scIL-12, or PBS either weekly or 2x weekly for a maximum of 5 doses. Mice were monitored 2x weekly for tumor growth as measured by digital calipers (Vernier) taking tumor volume to be $\mathrm{L} * \hat{\mathrm{W}} 2 * 1 / 2$ where $\mathrm{L}$ is the longest diameter and $\mathrm{W}$ the shortest diameter of the tumor. Mice were euthanized when volume exceeded $1000 \mathrm{~mm}^{3}$. For combined toxicity/efficacy studies mice were monitored daily for weight changes over the dosing period and serum was collected 24 hours after the first and last dose and analyzed for IFN- $\gamma$ and IL-12 content via ELISA (Peprotech) in addition to the above.

MC38 single tumor studies (Fig 6): (PBS, PLE-NPs, IL-12 n=10; PLE-IL-12-NPs $5 \mu \mathrm{g}$ weekly and $2 \mathrm{x}$ weekly $\mathrm{n}=8$; PLE-IL-12-NPs $7.5 \mu \mathrm{g} \mathrm{n}=7$ across multiple studies)

HM-1 single tumor studies (Fig 6): (PBS, PLE-NPs, and PLE-IL-12-NPs n=10; IL-12 n=9 across multiple studies)

High dose MC38 single tumor studies (Fig 7): (PLE-NPs n=3; $25 \mu \mathrm{g}$ PLE-IL-12-NP, $25 \mu \mathrm{g}$ IL-12, $50 \mu \mathrm{g}$ IL-12 n=5; $50 \mu \mathrm{g}$ PLE-IL-12-NPs n=4)

\section{Abscopal Response}

C57B1/6 mice were injected subcutaneously with 5E05 cells in 1:1 PBS:matrigel mixture on the right flank and 1E05 cells in 1:1 PBS:matrigel mixture of the left flank. Tumors were allowed to establish for 6 days prior to treatment. Mice were treated intratumorally with PLE-IL-12-NPs, dose matched soluble IL-12, or PBS weekly for 5 doses in the right tumor only. Mice were monitored $2 \mathrm{x}$ weekly for tumor growth as measured by digital calipers (Vernier) taking tumor volume to be $\mathrm{L} * \hat{\mathrm{W}} 2 * 1 / 2$ where $\mathrm{L}$ is the longest diameter and $\mathrm{W}$ the shortest diameter of the tumor. Mice were euthanized when volume exceeded $1000 \mathrm{~mm}^{3}$. (PBS, PLE-IL-12-NPs n=9; IL-12 n=8)

\section{Statistical Analysis}

GraphPad PRISM 5 was used to perform statistical analyses. Multiple comparisons were performed using multiple $t$ tests, one-way ANOVA, or two-way ANOVA followed by posthoc tests as indicated in figures. 


\title{
Data Availability
}

The data for this study are available within the article, with additional data available in the Supporting Information.

\section{Supplementary Material}

Refer to Web version on PubMed Central for supplementary material.

\section{ACKNOWLEDGEMENTS}

The authors also thank the MIT Koch Institute Swanson Biotechnology Center, which is supported by the Koch Institute Core Grant P30-CA14051 from the NCI, for the use of facilities and specifically the High Throughput Screening, Flowcytometry, Microscopy, and Animal Imaging and Preclinical Testing core facilities. We thank E. Dreaden for the helpful discussions and advice.

\begin{abstract}
This work was supported by the US Department of Defense Congressionally Directed Medical Research Programs (PTH, Teal Innovator Award W81XWH-13-1-0151), the Bridge Project a partnership between the Koch Institute for Integrative Cancer Research at MIT and the Dana-Farber/Harvard Cancer Center, NIH (1-R01-CA235375-01A1), the Marble Center for Cancer Nanomedicine, and a Cancer Center Support (core) Grant P30-CA14051 from the National Cancer Institute, the Koch Institute's Marble Center for Cancer Nanomedicine Fellowship (SGS), Sloan UCEM Fellowship (SC), the Siebel Scholars Fellowship (SC), the National Science Foundation (SC, GFRP 1122374), the MIT Undergraduate Research Opportunity Office (CN), the Frost Environmental Science/Studies Fund EN34770 (BTN), Ronald E. McNair Post-Baccalaureate Achievement Program GR26419 (BTN), and NIH interdepartmental biotechnology training program 5T32GM008334-30 (AEB). Resources were provided in part by the Koch Institute Support Grant (P30-CA14051) from the National Cancer Institute and the MIT MRSEC Shared Experimental Facilities Grant (DMR-0819762) from the National Science Foundation.
\end{abstract}

\section{REFERENCES}

1. Chen DS, Mellman I. Oncology Meets Immunology: The Cancer-Immunity Cycle. Immunity. 2013,39,1-10. [PubMed: 23890059]

2. Chen DS, Mellman I. Elements of Cancer Immunity and the Cancer-Immune Set Point. Nature. 2017,541,321-30. [PubMed: 28102259]

3. Bindea G, Mlecnik B, Angell HK, Galon J. The Immune Landscape of Human Tumors: Implications for Cancer Immunotherapy. OncoImmunology. 2014,3,e27456. [PubMed: 24800163]

4. Galon J, Pages F, Marincola FM, Angell HK, Thurin M, Lugli A, Zlobec I, Berger A, Bifulco C, Botti G, Tatangelo F, Britten CM, Kreiter S, Chouchane L, Delrio P, Arndt H, Asslaber M, Maio M, Masucci GV, Mihm M, et al. Cancer Classification Using the Immunoscore: A Worldwide Task Force. J. Transl. Med 2012,10,1-10. [PubMed: 22214470]

5. Pages F, Mlecnik B, Marliot F, Bindea G, Ou FS, Bifulco C, Lugli A, Zlobec I, Rau TT, Berger MD, Nagtegaal ID, Vink-Borger E, Hartmann A, Geppert C, Kolwelter J, Merkel S, Grutzmann R, Van den Eynde M, Jouret-Mourin A, Kartheuser A, et al. International Validation of the Consensus Immunoscore for the Classification of Colon Cancer: A Prognostic and Accuracy Study. Lancet. 2018,391,2128-2139. [PubMed: 29754777]

6. Trinchieri G Interleukin-12: A Proinflammatory Cytokine With Immunoregulatory Functions That Bridge Innate Resistance and Antigen-Specific Adaptive Immunity. Annu. Rev. Immunol 1995,13,251-276. [PubMed: 7612223]

7. Robertson MJ, Ritz J. Interleukin 12: Basic Biology and Potential Applications in Cancer Treatment. Oncologist. 1996,1,88-97. [PubMed: 10387973]

8. Trinchieri G; Scott P. Interleukin-12: Basic Principles and Clinical Applications In Redirection of Th1 and Th2 Responses; Coffman RL, Romagnani S, Eds; Springer: Berlin, Heidelberg, 1999; p5778.

9. Colombo MP, Trinchieri G. Interleukin-12 in Anti-Tumor Immunity and Immunotherapy. Cytokine Growth Factor Rev. 2002,13,155-168. [PubMed: 11900991] 
10. Brunda MJ, Luistro L, Warrier RR, Wright RB, Hubbard BR, Murphy M, Wolf SF, Gately M. Antitumor and Antimetastatic Activity of Interleukin 12 against Murine Tumors. J. Exp. Med 1993,178,1223-1230. [PubMed: 8104230]

11. Atkins MB, Robertson MJ, Gordon M, Lotze MT, DeCoste M, DuBois JS, Ritz J, Sandler AB, Edington HD, Garzone PD. Phase I Evaluation of Intravenous Recombinant Human Interleukin 12 in Patients with Advanced Malignancies. Clin. Cancer Res 1997,3,409-417. [PubMed: 9815699]

12. van Herpen CM, Huijbens R, Looman M, de Vries J, Marres H, van de Ven J, Hermsen R, Adema GJ, De Mulder PH. Pharmacokinetics and Immunological Aspects of a Phase Ib Study with Intratumoral Administration of Recombinant Human Interleukin-12 in Patients With Head and Neck Squamous Cell Carcinoma: A Decrease of T-Bet in Peripheral Blood Mononuclear Cells. Clin. Cancer Res 2003,9,2950-2956. [PubMed: 12912941]

13. Portielje JE, Kruit WH, Schuler M, Beck J, Lamers CH, Stoter G, Huber C, de Boer-Dennert M, Rakhit A, Bolhuis RL. Phase I Study of Subcutaneously Administered Recombinant Human Interleukin 12 in Patients with Advanced Renal Cell Cancer. Clin. Cancer Res 1999,5,3983-3989. [PubMed: 10632329]

14. Egilmez NK, Jong YS, Sabel MS, Jacob JS, Mathiowitz E, Bankert RB. In Situ Tumor Vaccination with Interleukin-12-Encapsulated Biodegradable Microspheres: Induction of Tumor Regression and Potent Antitumor Immunity. Cancer Res. 2000,60,3832-3837. [PubMed: 10919657]

15. Hill HC, Conway TF, Sabel MS, Jong YS, Mathiowitz E, Bankert RB, Egilmez NK. Cancer Immunotherapy with Interleukin 12 and Granulocyte-Macrophage Colony-Stimulating FactorEncapsulated Microspheres: Coinduction of Innate and Adaptive Antitumor Immunity and Cure of Disseminated Disease. Cancer Res. 2002,62,7254-7263. [PubMed: 12499267]

16. Sabel MS, Hill H, Jong YS, Mathiowitz E, Bankert RB, Egilmez NK. Neoadjuvant Therapy with Interleukin-12-Loaded Polylactic Acid Microspheres Reduces Local Recurrence and Distant Metastases. Surgery. 2001,130,470-478. [PubMed: 11562672]

17. Sabel MS, Arora A, Su G, Mathiowitz E, Reineke JJ, Chang AE. Synergistic Effect of Intratumoral IL-12 and TNF-a Microspheres: Systemic Anti-Tumor Immunity Is Mediated by Both CD8+ CTL and NK Cells. Surgery. 2007,142,749-760. [PubMed: 17981196]

18. Smith SG, Koppolu Bp, Ravindranathan S, Kurtz SL, Yang L, Katz MD, Zaharoff DA. Intravesical Chitosan/Interleukin-12 Immunotherapy Induces Tumor-Specific Systemic Immunity against Murine Bladder Cancer. Cancer Immunol. Immunother 2015,64,689-696. [PubMed: 25754122]

19. Vo JL, Yang L, Kurtz SL, Smith SG, Koppolu Bp, Ravindranathan S, Zaharoff DA. Neoadjuvant Immunotherapy with Chitosan and Interleukin-12 to Control Breast Cancer Metastasis. OncoImmunology. 2014,3,e968001. [PubMed: 25964864]

20. Yang L, Zaharoff DA. Role of Chitosan Co-Formulation in Enhancing Interleukin-12 Delivery and Antitumor Activity. Biomaterials. 2013,34,3828-3836. [PubMed: 23453060]

21. Lai I, Swaminathan S, Baylot V, Mosley A, Dhanasekaran R, Gabay M, Felsher DW. Lipid Nanoparticles That Deliver IL-12 Messenger RNA Suppress Tumorigenesis in MYC OncogeneDriven Hepatocellular Carcinoma. J. Immunother. Cancer 2018,6,125. [PubMed: 30458889]

22. Thaker PH, Bradley WH, Leath CA, Gunderson CC, Borys N, Musso L, Anwer K, Alvarez RD. Phase I Study of the Safety and Activity of Formulated IL-12 Plasmid Administered Intraperitoneally in Combination with Neoadjuvant Chemotherapy in Patients With Newly Diagnosed Advanced-Stage Ovarian Cancer. J. Clin. Oncol 2019,37,2-2.

23. Xu Q, Guo L, Gu X, Zhang B, Hu X, Zhang J, Chen J, Wang Y, Chen C, Gao B. Prevention of Colorectal Cancer Liver Metastasis by Exploiting Liver Immunity via Chitosan-TPP/Nanoparticles Formulated with IL-12. Biomaterials. 2012,33,3909-3918. [PubMed: 22374455]

24. Shimizu T, Kishida T, Hasegawa U, Ueda Y, Imanishi J, Yamagishi H, Akiyoshi K, Otsuji E, Mazda O. Nanogel DDS Enables Sustained Release of IL-12 for Tumor Immunotherapy. Biochem. Biophys. Res. Commun 2008,367,330-335. [PubMed: 18158918]

25. Colombo MP, Vagliani M, Spreafico F, Parenza M, Chiodoni C, Melani C, Stoppacciaro A. Amount of Interleukin 12 Available at the Tumor Site Is Critical for Tumor Regression. Cancer Res. 1996,56,2531-2534. [PubMed: 8653692]

26. Decher G Fuzzy Nanoassemblies: Toward Layered Polymeric Multicomposites. Science. 1997,277,1232-1237. 
27. Hammond PT. Form and Function in Multilayer Assembly: New Applications at the Nanoscale. Adv. Mater 2004,16,1271-1293.

28. Shaillender M, Luo RC, Venkatraman SS, Neu B. Layer-by-Layer Microcapsules Templated on Erythrocyte Ghost Carriers. Int. J. Pharm 2011,415,211-217. [PubMed: 21699968]

29. Dreaden EC, Kong YW, Morton SW, Correa S, Choi KY, Shopsowitz KE, Renggli K, Drapkin R, Yaffe MB, Hammond PT. Tumor-Targeted Synergistic Blockade of MAPK and PI3K from a Layer-by-Layer Nanoparticle. Clin. Cancer Res 2015,21,4410-4419. [PubMed: 26034127]

30. Dreaden EC, Morton SW, Shopsowitz KE, Choi J-H, Deng ZJ, Cho N-J, Hammond PT. Bimodal Tumor-Targeting from Microenvironment Responsive Hyaluronan Layer-by-Layer (LbL) Nanoparticles. ACS Nano. 2014,8,8374-8382. [PubMed: 25100313]

31. Hammond PT. Engineering Materials Layer-by-Layer: Challenges and Opportunities in Multilayer Assembly. AIChE J. 2011,57,2928-2940.

32. Correa S, Choi KY, Dreaden EC, Renggli K, Shi A, Gu L, Shopsowitz KE, Quadir MA, Ben-Akiva E, Hammond PT. Highly Scalable, Closed-Loop Synthesis of Drug-Loaded, Layer-by-Layer Nanoparticles. Adv. Funct. Mater 2016,26,991-1003. [PubMed: 27134622]

33. Correa S, Dreaden EC, Gu L, Hammond PT. Engineering Nanolayered Particles for Modular Drug Delivery. J. Controlled Release 2016,240,364-386.

34. Dang X, Gu L, Qi J, Correa S, Zhang G, Belcher AM, Hammond PT. Layer-by-Layer Assembled Fluorescent Probes in the Second Near-Infrared Window for Systemic Delivery and Detection of Ovarian Cancer. Proc. Natl. Acad. Sci. U. S. A 2016,113,5179-5184. [PubMed: 27114520]

35. Deng ZJ, Morton SW, Ben-Akiva E, Dreaden EC, Shopsowitz KE, Hammond PT. Layer-by-Layer Nanoparticles for Systemic Codelivery of an Anticancer Drug and siRNA for Potential TripleNegative Breast Cancer Treatment. ACS Nano. 2013,7,9571-9584. [PubMed: 24144228]

36. Morton SW, Lee MJ, Deng ZJ, Dreaden EC, Siouve E, Shopsowitz KE, Shah NJ, Yaffe MB, Hammond PT. A Nanoparticle-Based Combination Chemotherapy Delivery System for Enhanced Tumor Killing by Dynamic Rewiring of Signaling Pathways. Sci. Signaling 2014; 7,ra44.

37. Morton SW, Poon Z, Hammond PT. The Architecture and Biological Performance of Drug-Loaded LbL Nanoparticles. Biomaterials. 2013,34,5328-5335. [PubMed: 23618629]

38. Lieschke GJ, Mulligan RC. Bioactive Fusion Proteins Comprising the p35 and p40 Subunits of IL-12. US5891680A, 1999.

39. Colletier J-P, Chaize B, Winterhalter M, Fournier D. Protein Encapsulation in Liposomes: Efficiency Depends on Interactions between Protein and Phospholipid Bilayer. BMC Biotechnol. 2002,2,1-8. [PubMed: 11818033]

40. Johnston AP, Cortez C, Angelatos AS, Caruso F. Layer-by-Layer Engineered Capsules and Their Applications. Curr. Opin. Colloid Interface Sci 2006,11,203-209.

41. Correa S, Boehnke N, Barberio AE, Deiss-Yehiely E, Shi A, Oberlton B, Smith SG, Zervantonakis IK, Dreaden EC, Brugge JS, Hammond PT. Tuning Nanoparticle Interactions with Ovarian Cancer through Layer-by-Layer Modification of Surface Chemistry. ACS Nano. 2020,14,2224-2237. [PubMed: 31971772]

42. Gu L, Deng ZJ, Roy S, Hammond PT. A Combination RNAi-Chemotherapy Layer-by-Layer Nanoparticle for Systemic Targeting of KRAS/P53 with Cisplatin to Treat Non-Small Cell Lung Cancer. Clin. Cancer Res 2017,23,7312-7323. [PubMed: 28912139]

43. Cohen K, Emmanuel R, Kisin-Finfer E, Shabat D, Peer D. Modulation of Drug Resistance in Ovarian Adenocarcinoma Using Chemotherapy Entrapped in Hyaluronan-Grafted Nanoparticle Clusters. ACS Nano. 2014,8,2183-2195. [PubMed: 24494862]

44. Ganesh S, Iyer AK, Gattacceca F, Morrissey DV, Amiji MM. In Vivo Biodistribution of siRNA and Cisplatin Administered Using CD44-Targeted Hyaluronic Acid Nanoparticles. J. Controlled Release 2013,172,699-706.

45. Choi KY, Yoon HY, Kim J-H, Bae SM, Park R-W, Kang YM, Kim I-S, Kwon IC, Choi K, Jeong SY. Smart Nanocarrier Based on PEGylated Hyaluronic Acid for Cancer Therapy. ACS Nano. 2011,5,8591-8599. [PubMed: 21967065]

46. Hasan M, Najjam S, Gordon MY, Gibbs RV, Rider CC. IL-12 Is a Heparin-Binding Cytokine. J. Immunol 1999,162,1064-1070. [PubMed: 9916734] 
47. Abiko K, Matsumura N, Hamanishi J, Horikawa N, Murakami R, Yamaguchi K, Yoshioka Y, Baba T, Konishi I, Mandai M. IFN- $\gamma$ From Lymphocytes Induces PD-L1 Expression and Promotes Progression of Ovarian Cancer. Br. J. Cancer 2015,112,1501-1509. [PubMed: 25867264]

48. Peng J, Hamanishi J, Matsumura N, Abiko K, Murat K, Baba T, Yamaguchi K, Horikawa N, Hosoe Y, Murphy SK. Chemotherapy Induces Programmed Cell Death-Ligand 1 Overexpression via the Nuclear Factor- $\kappa$ B to Foster an Immunosuppressive Tumor Microenvironment in Ovarian Cancer. Cancer Res. 2015,75,5034-5045. [PubMed: 26573793]

49. Ott PA, Hodi FS, Kaufman HL, Wigginton JM, Wolchok JD. Combination Immunotherapy: A Road Map. J. Immunother. Cancer 2017,5,16. [PubMed: 28239469]

50. Schmidt C Combinations on Trial. Nature. 2017;552:S67-S69.

51. Weiss JM, Subleski JJ, Wigginton JM, Wiltrout RH. Immunotherapy of Cancer by IL-12-Based Cytokine Combinations. Expert Opin. Biol. Ther 2007,7,1705-1721. [PubMed: 17961093]

52. Rothschilds AM, Wittrup KD. What, Why, Where, and When: Bringing Timing to ImmunoOncology. Trends Immunol. 2019,40,12-21. [PubMed: 30545676]

53. Stewart JCM. Colorimetric Determination of Phospholipids With Ammonium Ferrothiocyanate. Anal. Biochem 1980,104,10-14. [PubMed: 6892980]

54. Liposome: Encapsula's Scientific Blog. www.liposomes.org/2009/01/number-of-lipid-moleculesper-liposome.html (March 29, 2020), The Number of Lipid Molecules per Liposome. 


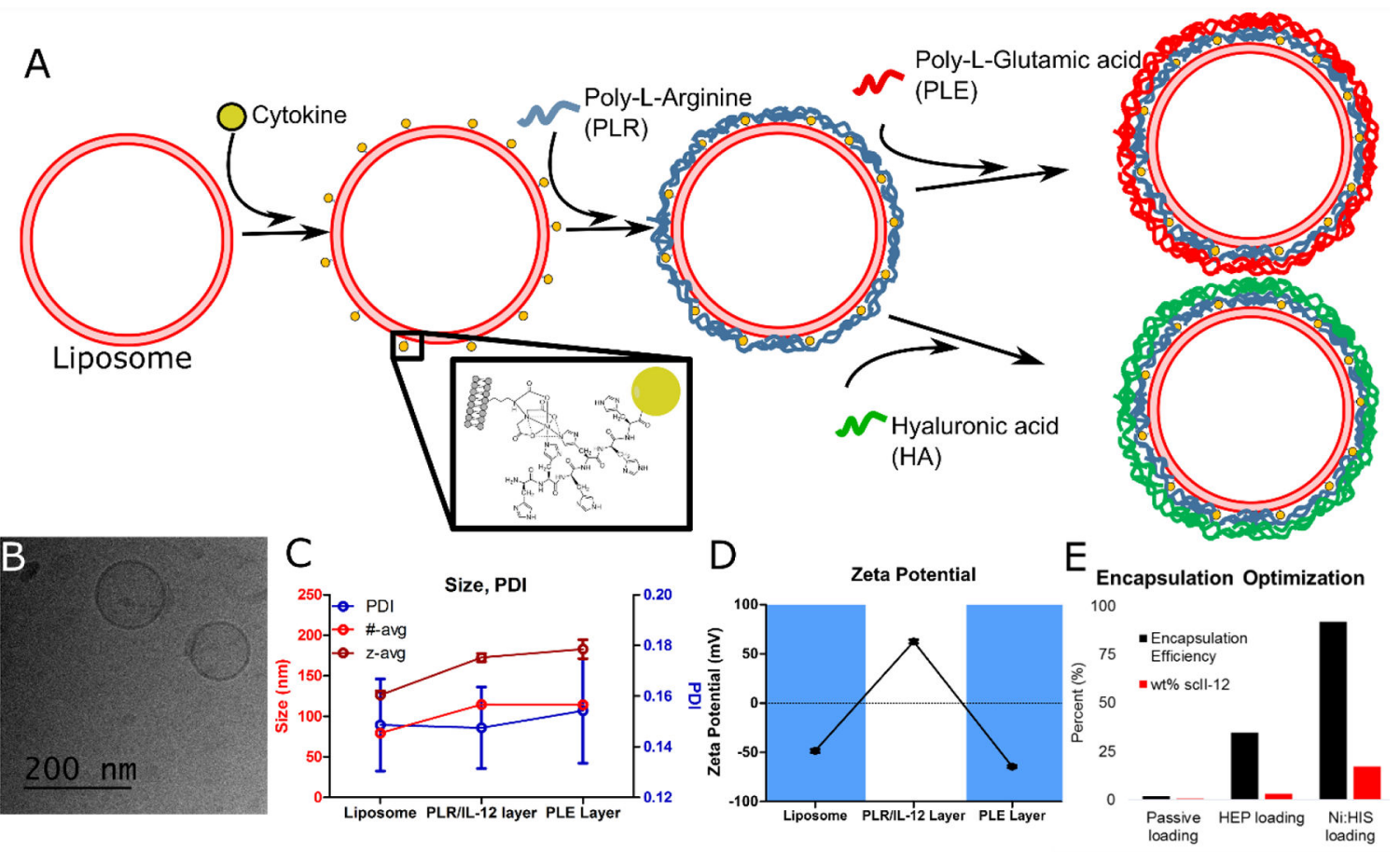

Figure 1.

ScIL-12 is formulated into monodisperse LbL-NP. A, Schematic of layer-by-layer buildup of particle and cytokine attachment. B, cryoEM image of LbL-NPs shows layered liposome structures $80-120 \mathrm{~nm}$ in diameter. C, Dynamic light scattering measurements show effective layering of particles by diameter increase and $\mathbf{D}$, charge reversal through layering process, resulting in $\sim 110 \mathrm{~nm}$ negatively charged particles. Data presented for PLE terminal polyanion layer particles, other external polymers showed similar results by DLS. E, Encapsulation of scIL-12 in LbL-NPs as measured by ELISA for different encapsulation techniques (passive, heparin layering interaction (HEP), Ni His tag interaction (Ni:His)). 

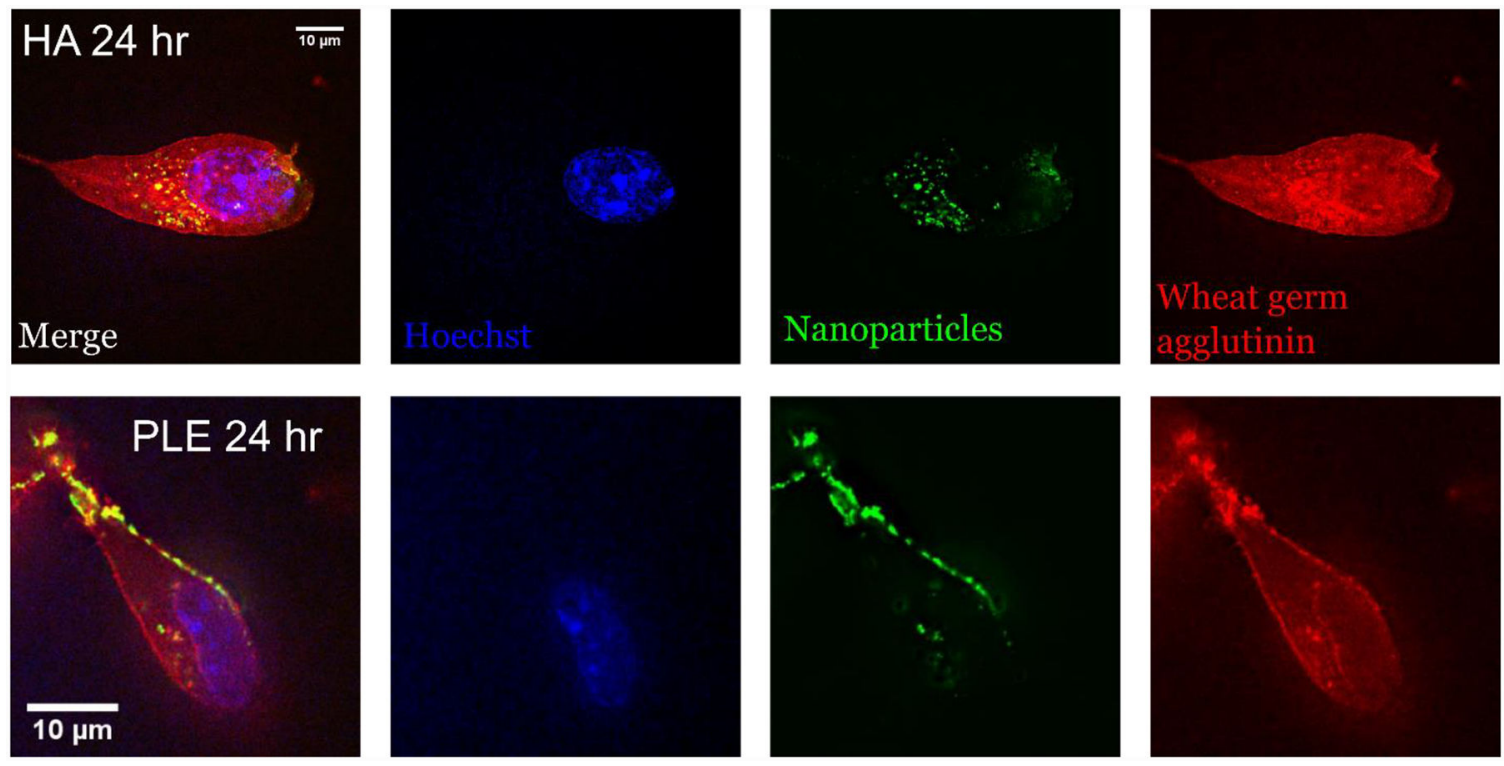

Figure 2.

LbL-NPs demonstrate differential interactions with cancer cells based on surface chemistry. Fluorescence imaging of MC38 tumor cells incubated with fluorescent carboxy-modified latex core LbL-NPs with either HA or PLE terminal layers for 24 hours. Blue indicates Hoechst nuclear stain, green is LbL-NP fluorescence, red marks wheat germ agglutinin membrane stain. 


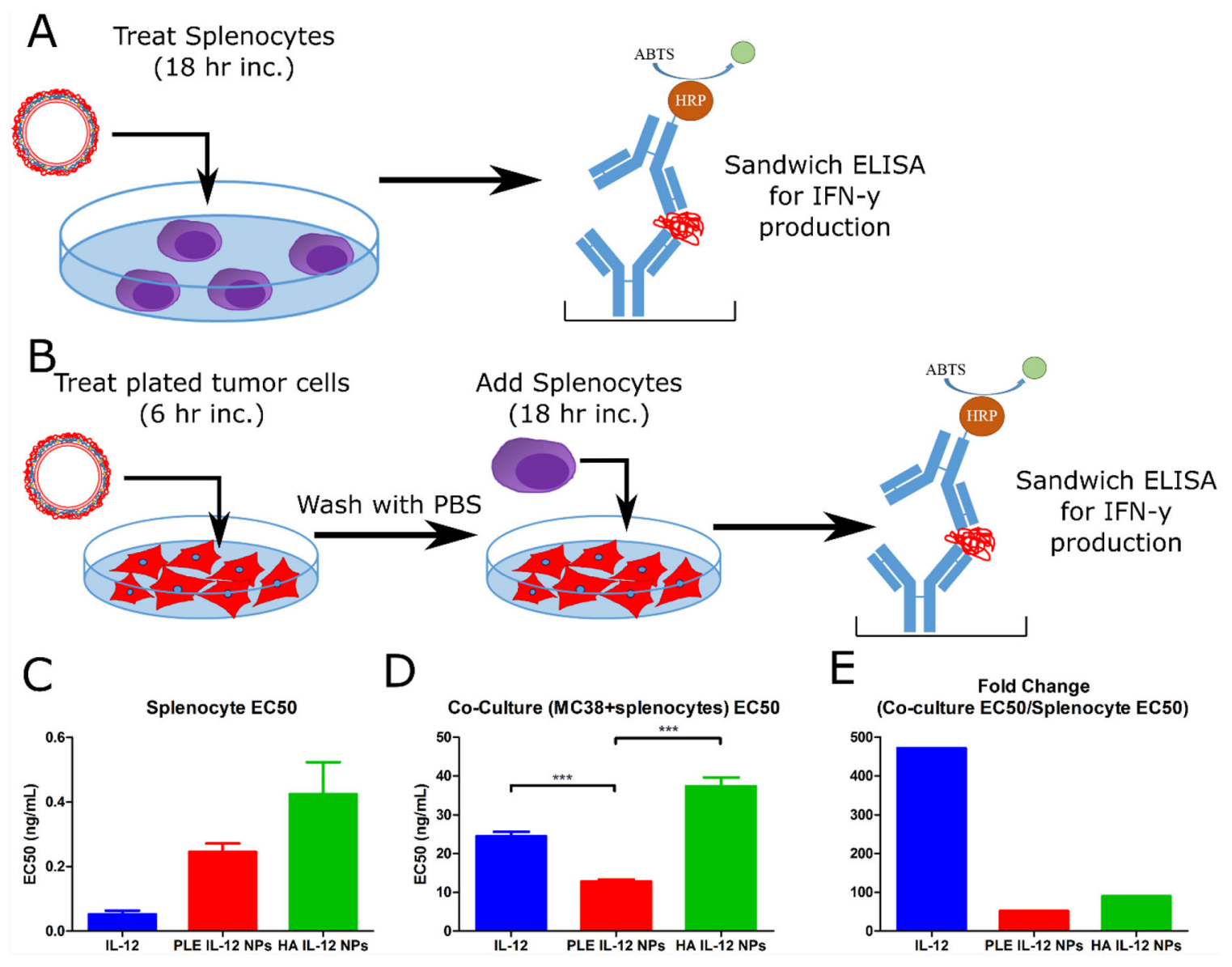

Figure 3.

LbL-IL-12-NPs demonstrate enhanced efficacy in vitro. A, Schematic of in vitro efficacy test on splenocyte culture. $\mathbf{B}$, Schematic of in vitro efficacy test in tumor mimic co-culture. C, EC50 of IFN- $\gamma$ response of splenocytes treated with varying IL-12 therapies from A. D, EC50 (+SEM) of IFN- $\gamma$ response of co-cultured MC38 cells and splenocytes treated with varying IL-12 therapies from B. *** indicated $\mathrm{p}<.001$ calculated by one-way ANOVA with Bonferroni post hoc test across all groups. E, Fold change of activity between C and D. 

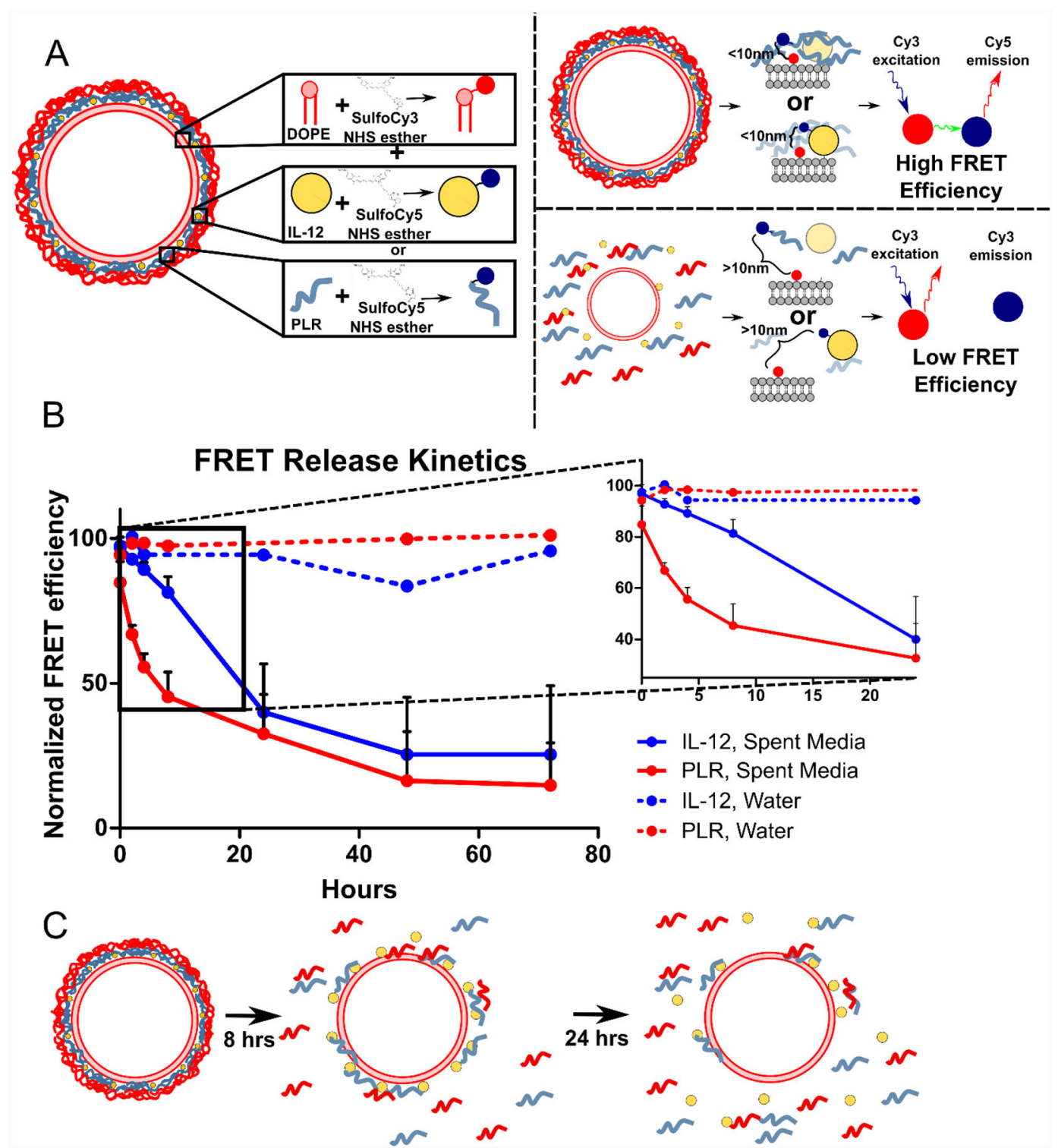

Figure 4.

Activity mechanism from PLE-IL-12-NPs. A, Schematic of NP structure for FRET kinetic release studies (left). FRET readouts for intact NPs (right, top) and degraded NPs (right, bottom) B, Normalized FRET efficiency overtime for NPs. NPs were tested under two media conditions: water (storage media, dashed curves) and spent media (MC38 media culture on cells for at least 48 hours as an in vivo mimic, solid curves). FRET efficiencies were measured for the erosion of the polymer layers as measured by FRET pairing with the acceptor fluorophore on PLR (blue) and release of IL-12 as measured by FRET pairing with the acceptor fluorophore on IL-12 (red). Inset shows the first 24 hours in greater detail. C, schematic of particle break down as indicated by release data from B. 

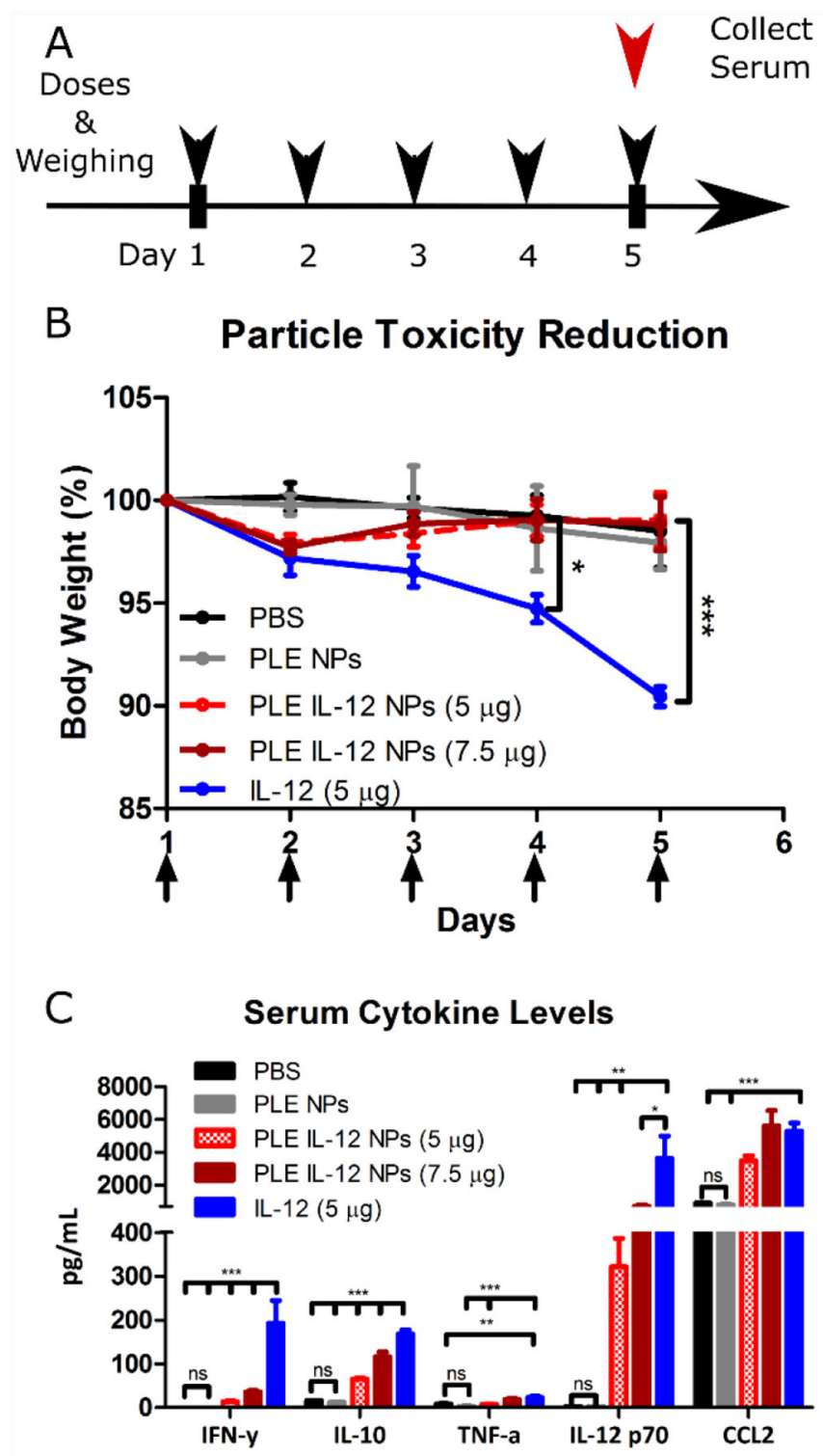

Figure 5.

PLE IL-12 NPs reduce toxicity of IL-12 therapy in vivo. A, Schematic of experimental design B, Body weight change (Mean+SEM) of healthy animals treated as indicated subcutaneously (PLE IL-12 NPs $5 \mu \mathrm{g} \mathrm{n}=5, \mathrm{n}=3$ all other groups). * indicates $\mathrm{p}<.05$, *** indicates $\mathrm{p}<.001$ as measured by 2-way ANOVA with Bonferroni post-hoc test across all groups. C, Cytokine response (Mean+SEM) in serum taken after dose 5 from $\mathrm{B}$ as measured by multiplexed assay. * indicates $\mathrm{p}<.05$, ** indicates $\mathrm{p}<.01, * * *$ indicates $\mathrm{p}<.001$ as measured by One way ANOVA on individual cytokine groups with Bonferroni post-hoc test

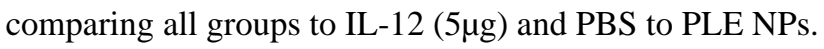



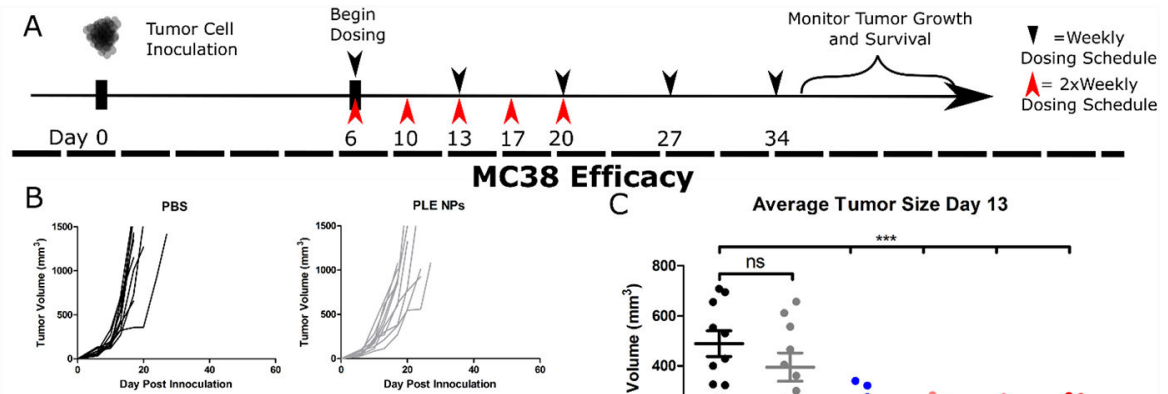

C Average Tumor Size Day 13
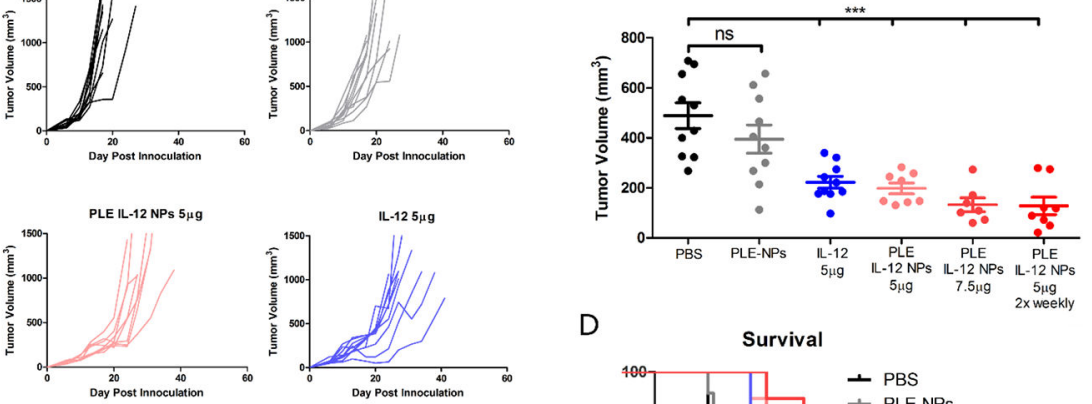

$\mathrm{D}$
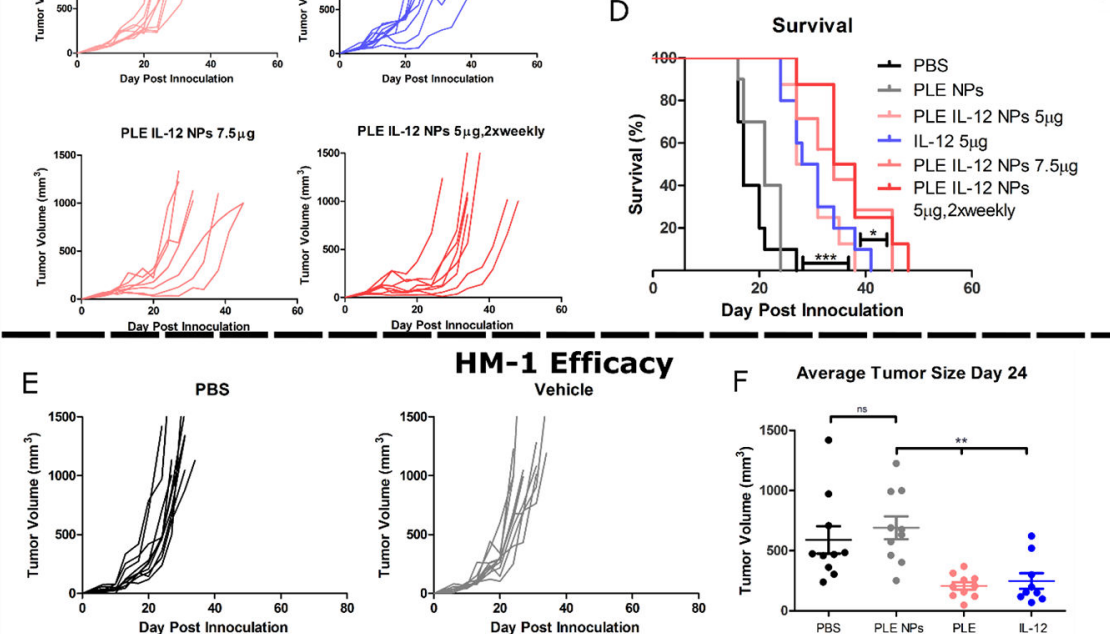

PLE IL-12 NPs $5 \mu \mathrm{g}, 2 \times w e e k l y$
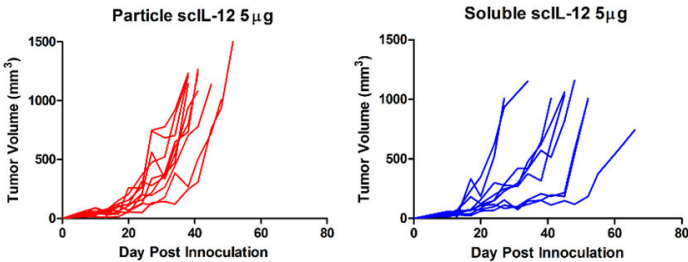

F Average Tumor Size Day 24
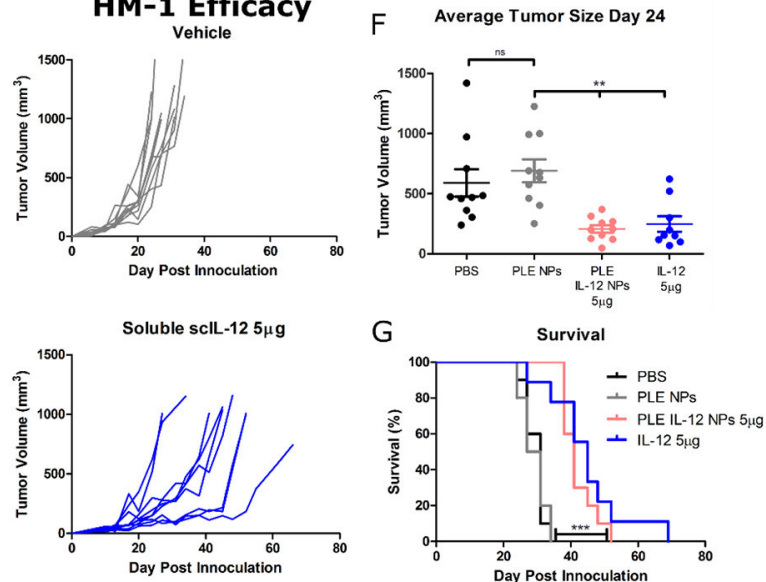

Figure 6.

PLE IL-12 NPs maintain efficacy against MC38 (A-D) and HM-1 (E-G) tumors in vivo. A, Study design. B, MC38 tumor volume $\left(\mathrm{mm}^{3}\right)$ of indicated treatments. All animals dosed 5 times given weekly intratumorally unless otherwise noted beginning on day 6. (PBS, PLENPs, IL-12 n=10; PLE-IL-12-NPs $5 \mu \mathrm{g}$ weekly and 2x weekly $n=8$; PLE-IL-12-NPs $7.5 \mu \mathrm{g}$ $\mathrm{n}=7$ across multiple studies) $\mathbf{C}$, Mean volume (+SEM) from B on day 13 for each group. *** indicates $\mathrm{p}<.001$ as measured by one-way ANOVA using Bonferroni post-hoc test on all pairs of data. D, Survival curves of groups from B and C. * indicates $\mathrm{p}<.05$, *** indicates $\mathrm{p}<.0001$ as measured by Log-rank tests between groups. E, HM-1 tumor volume $\left(\mathrm{mm}^{3}\right)$ of indicated treatments. All animals dosed 5 times given weekly intratumorally beginning on day 6. (PBS, PLE-NPs, and PLE-IL-12-NPs $n=10$; IL-12 $\mathrm{n}=9$ across multiple studies) $\mathbf{F}$, Mean volume (+SEM) from $\mathrm{E}$ on day 24 for each group. ** indicates $\mathrm{p}<.01$ as measured by 
one-way ANOVA using Bonferroni post-hoc test on all pairs of data. G, Survival curves of groups from $\mathrm{E}$ and $\mathrm{F}$. *** indicates $\mathrm{p}<.0001$ as measured by Log-rank tests between groups. 
A
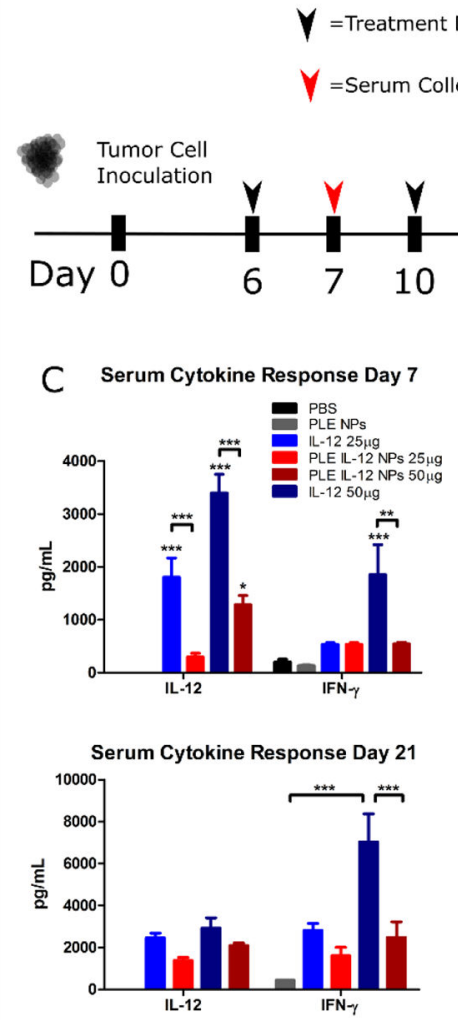

$\mathrm{E}$

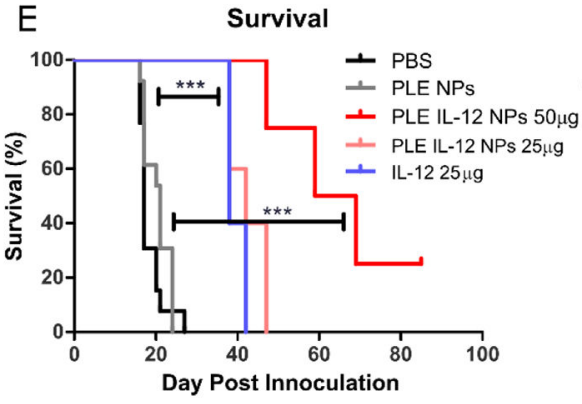

Monitor

Tumor Growth

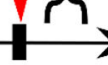

$17 \quad 20 \quad 21$

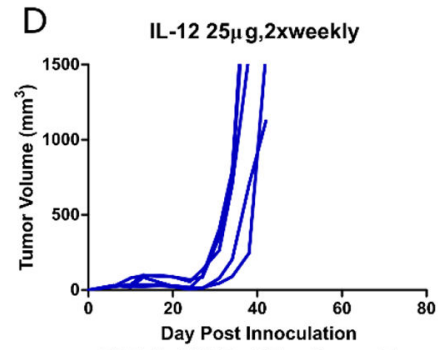

PLE IL-12 NPs 25 $\mathrm{g}$,2xweekly

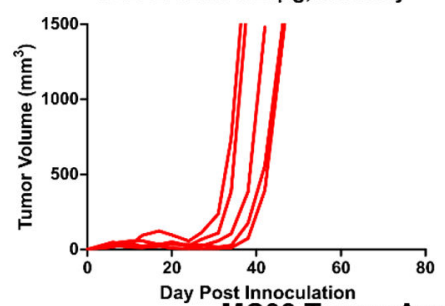

$F$

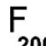
and Survival
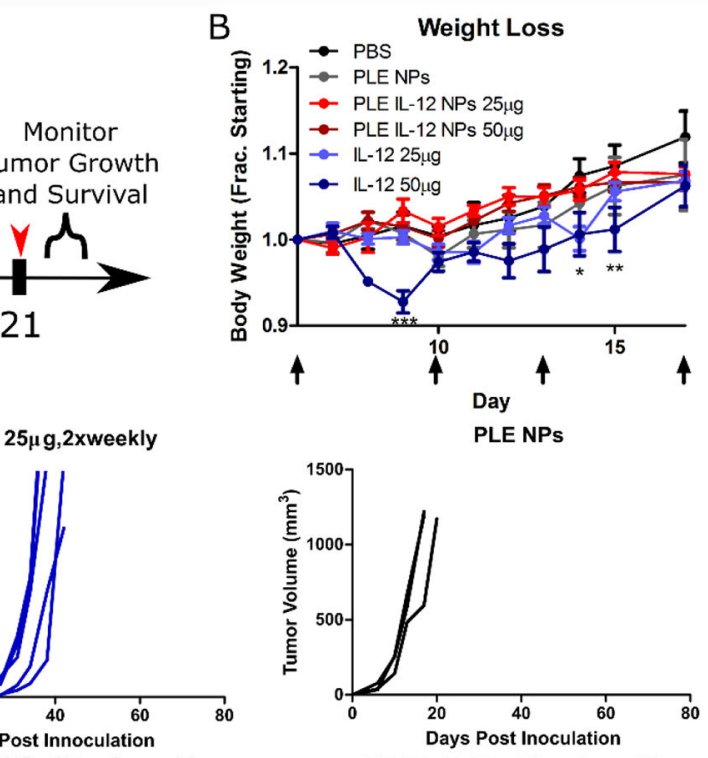

PLE IL-12 NPs $50 \mu \mathrm{g}, 2$ xweekly

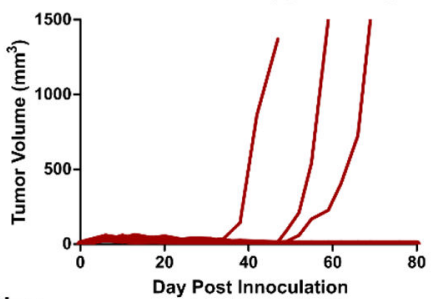

MC38 Tumor Avg Size

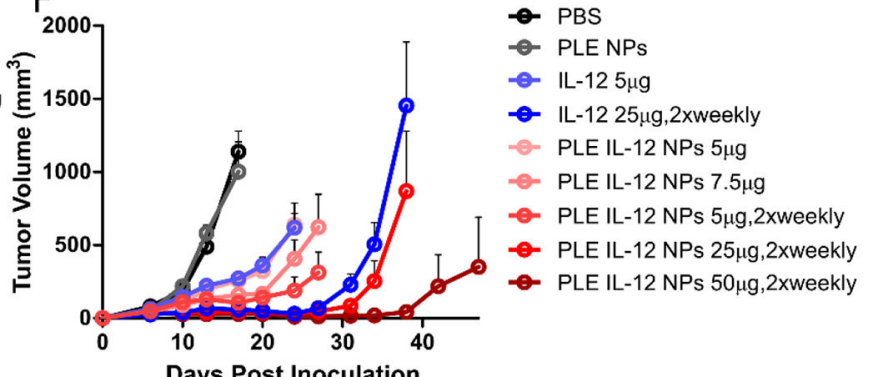

Figure 7.

Combined toxicity and efficacy at high doses demonstrates PLE IL-12 NPs are a safer, more effective IL-12 therapy. A, Schematic of study. C57B1/6 mice are inoculated with 5E06 MC38 cells on day 0 . Mice are treated twice weekly for 5 doses with serum collected $24 \mathrm{hrs}$ after the first and last dose. Animals are monitored throughout for tumor burden and weight change. (PLE-NPs n=3; $25 \mu \mathrm{g}$ PLE-IL-12-NP, $25 \mu \mathrm{g}$ IL-12, $50 \mu \mathrm{g}$ IL-12 n=5; $50 \mu \mathrm{g}$ PLEIL-12-NPs n=4) B, Weight change (Mean+SEM) over initial dosing period,* indicates $\mathrm{p}<.05, * *$ indicates $\mathrm{p}<.01, * * *$ indicates $\mathrm{p}<.001$ compared to PBS as measured by 2 -way ANOVA with Bonferroni post-hoc tests comparing all groups to PBS. C, Cytokine levels $($ Mean + SEM) in serum at indicated time points as measured by ELISA. * indicates $p<.05$, *** indicates $\mathrm{p}<.001$ as measured by 2 way ANOVA with Bonferroni post-hoc test comparing all pairs of groups. Comparisons are to PBS where not indicated otherwise. D, Tumor volumes of individual animals of indicated treatment groups. E, Survival curves of 
animals from treated and control groups (PBS and PLE NP combined over all studies). ** indicates $\mathrm{p}<.01, * * *$ indicates $\mathrm{p}<.001$ as measured by Log-rank tests between groups. $\mathbf{F}$, Average tumor volumes (mean $+\mathrm{SEM}$ ) over time for animals from figure 5B low dose

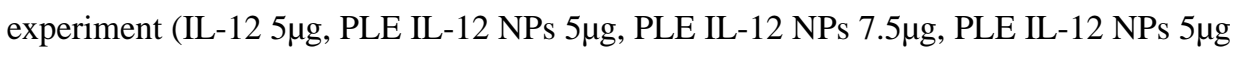

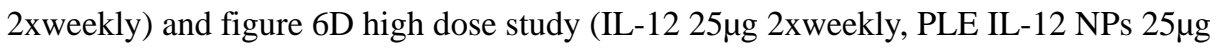

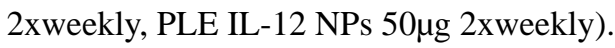



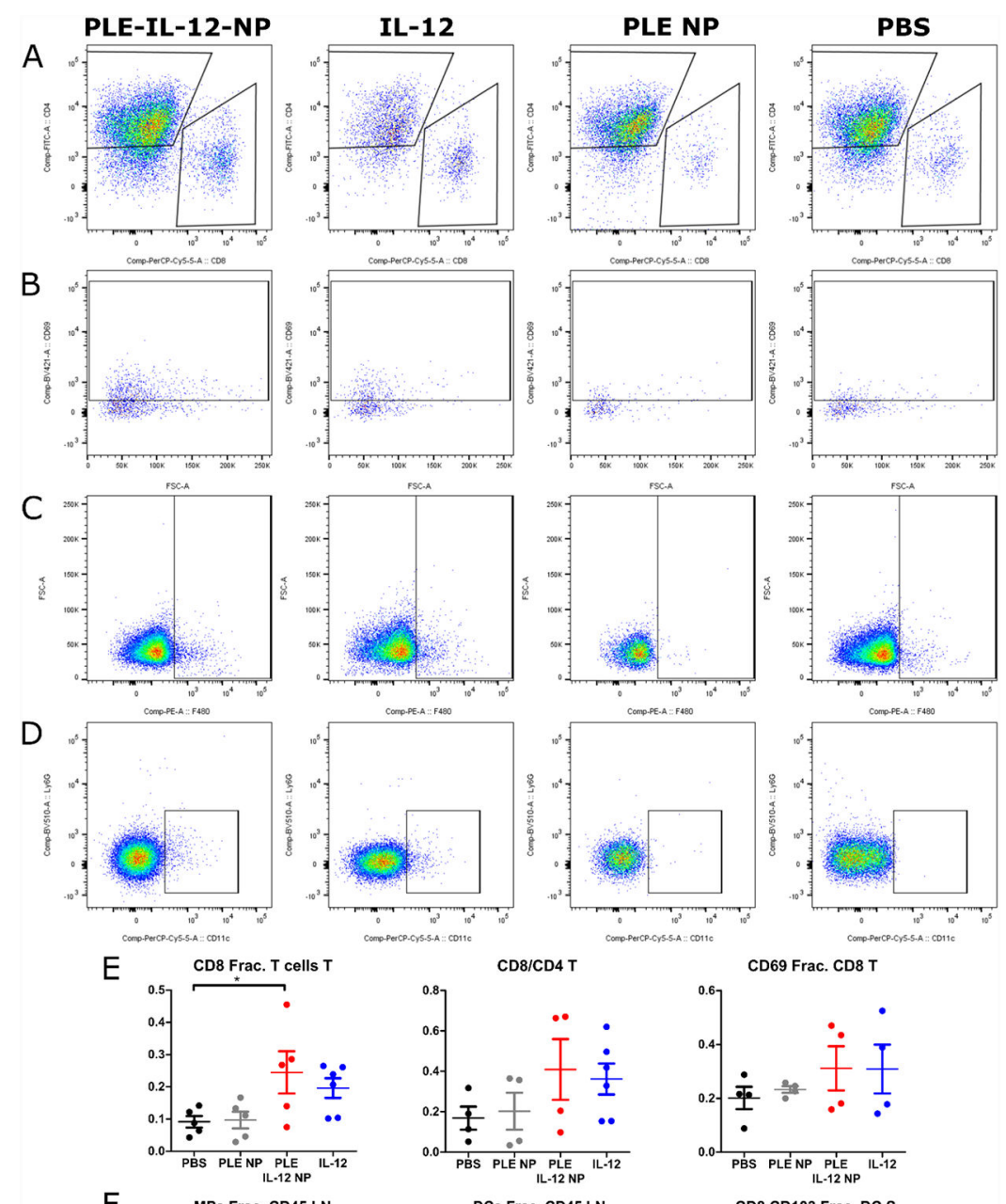

F
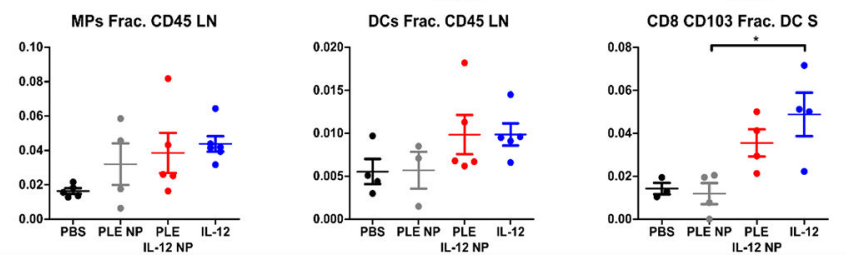

Figure 8.

Flowcytometry analysis shows IL-12 shifts immune populations towards an antitumoral response. A. CD3+ T cell gating for CD4 and CD8 T cells in different treatment groups (CD4 y-axis, CD8 x-axis). B. Degranulation of CD8+ T cells as measured by CD69 staining in the tumor. C. F4/80 staining of CD45+ cells in TDLNs. D. DC content as measured by CD11c+ staining in CD45+ cells in TDLNs. E. Quantification of chosen T cell populations from $\mathrm{A}$ and B. * indicates $\mathrm{p}<.05$ as measured by one way ANOVA with Dunnett posthoc test F. Quantification of chosen APC populations flowcytometry. ( $\mathrm{T}=$ tumor, $\mathrm{S}=$ spleen, $\mathrm{LN}=\mathrm{TDLN}$ ) 